\title{
Competition against peer-to-peer networks
}

Citation for published version (APA):

Herings, P. J. J., Peeters, R. J. A. P., \& Yang, M. (2008). Competition against peer-to-peer networks. METEOR, Maastricht University School of Business and Economics. METEOR Research Memorandum No. 020 https://doi.org/10.26481/umamet.2008020

Document status and date:

Published: 01/01/2008

DOI:

10.26481/umamet.2008020

Document Version:

Publisher's PDF, also known as Version of record

\section{Please check the document version of this publication:}

- A submitted manuscript is the version of the article upon submission and before peer-review. There can be important differences between the submitted version and the official published version of record.

People interested in the research are advised to contact the author for the final version of the publication, or visit the DOI to the publisher's website.

- The final author version and the galley proof are versions of the publication after peer review.

- The final published version features the final layout of the paper including the volume, issue and page numbers.

Link to publication

\footnotetext{
General rights rights.

- You may freely distribute the URL identifying the publication in the public portal. please follow below link for the End User Agreement:

www.umlib.nl/taverne-license

Take down policy

If you believe that this document breaches copyright please contact us at:

repository@maastrichtuniversity.nl

providing details and we will investigate your claim.
}

Copyright and moral rights for the publications made accessible in the public portal are retained by the authors and/or other copyright owners and it is a condition of accessing publications that users recognise and abide by the legal requirements associated with these

- Users may download and print one copy of any publication from the public portal for the purpose of private study or research.

- You may not further distribute the material or use it for any profit-making activity or commercial gain

If the publication is distributed under the terms of Article $25 \mathrm{fa}$ of the Dutch Copyright Act, indicated by the "Taverne" license above, 
P. Jean-Jacques Herings, Ronald Peeters, Michael Yang

Competition against peer-to-peer networks

$\mathrm{RM} / 08 / 020$

JEL code: L11, L82, L86

\section{METE@R}

Maastricht research school of Economics of TEchnology and ORganizations

Universiteit Maastricht

Faculty of Economics and Business Administration P.O. Box 616

NL - 6200 MD Maastricht

phone : ++31433883830

fax : ++31433884873 


\title{
Competition against peer-to-peer networks
}

\author{
P. Jean-Jacques Herings* Ronald Peeters* Michael Yang*
}

June 26, 2008

\begin{abstract}
In this paper, we consider the competition of providers of information products against P2P networks that offer illegal versions of the information products. Depending on the generic cost factor of downloading - incorporating factors including, among other things, the degree of legal enforcement of intellectual property rights - we find that the firm may employ pricing strategies to either deter the entry of a network or to accommodate it. In the latter case, we find that the equilibrium price moves in the opposite direction of the generic cost factor of downloading. This counter-intuitive result corresponds to a very subtle form of platform competition between the firm and the network. Furthermore, profits for the firm unambiguously decrease when the generic cost factor of downloading declines, whereas total welfare unambiguously increases. This implies that it may well be welfare enhancing to relax the legal enforcements of intellectual property rights.
\end{abstract}

JEL classification: L11; L82; L86.

Keywords: information products; the music industry; piracy; P2P (peer-to-peer) filesharing networks; network externalities; limit pricing; multi-platform competition.

\section{Introduction}

The tremendous amount of downloading and sharing of information goods on the internet in the past ten years are largely due to the wide spread and popularity of P2P (peer-topeer) file-sharing networks. The information content providers have, over the years, claimed that the usage of such file-sharing networks constitutes the primary cause of the steady decrease in their sales revenue, and resorted to law suits against the users of such networks on numerous occasions. Anti-piracy organizations have also contributed considerable work in helping the authorities reinforce intellectual property rights. Despite all their efforts, however, the file-sharing activities have, if anything, steadily increased in the past years, thanks to the increasing sophistication and non-tractability of today's file-sharing applications.

${ }^{*}$ Department of Economics, Maastricht University, P.O. Box 616, 6200 MD Maastricht, The Netherlands. E-mail: \{S.Yang, P.Herings, R.Peeters\}@algec.unimaas.nl. P. Jean-Jacques Herings would like to thank the Netherlands Organisation for Scientific Research (NWO) for financial support. Corresponding author: P. Herings. 
There have been numerous empirical studies about the music industry specifically. Blackburn (2004), Liebowitz (2004), Rob and Waldfogel (2006), and Zentner (2006) find that downloading (via P2P file-sharing networks) are at least partially responsible for the recent decrease in CD sales. Liebowitz (2006) concludes that file-sharing has clearly brought significant harm to the recording industry and labels his paper as "the end of the beginning stage of research" on the subject. In contrast, Oberholzer-Gee and Strumpf (2007) and Peitz and Waelbroek (2004) find little robust evidence that P2P has caused the decrease in CD sales for the recent years.

The theoretical literature related to this topic can be traced back to some early classical papers on piracy and network effects. ${ }^{1}$ Gayer and Shy (2003) studies piracy on P2P networks and the effect on the information product providers. In their paper, the authors investigate how publishers of digitally-stored products, including music, can utilize P2P to enhance sales of their product sold in the store. This result is mainly attributed to the positive consumptive externality ingredient in their model. Peitz and Waelbroek (2006) shows that under sufficient taste heterogeneity and product diversity, the positive effect of downloading on sales due to sampling may compensate the negative effect. The result is mainly due to the fact that there is information asymmetry between the buyers and the sellers of the product, and downloaded files can help buyers identify their favorite products more easily and hence encourage sales.

Although the question whether P2P harms or benefits the sales of CDs has received plenty of attention in the theoretical literature, most of the models assume a particular market structure, such as the co-existence of multi-platforms. Moreover, the models consider partially- and fully-served markets independently without analyzing what determines whether the market is served partially or fully. Neither do the models investigate the content provider's opportunity to deter entry of the network by application of a limit pricing policy. In general, little emphasis has been put on the strategic behavior of the publishing firm in terms of price competition against $\mathrm{P} 2 \mathrm{P}$, the resulting equilibrium market structures, the firm's profit, the consumers' surplus and the total welfare. These are the issues the present paper deals with.

We consider two different forms of information content products: the (original) physical form and the (illegal) digital form. These two forms, although essentially providing similar contents, differ in numerous ways. The physical form of the product is sold by the firm in the store as CDs or DVDs, usually accompanied by appealing packaging and complementary booklets. The digital form, which is shared on the internet via P2P networks, contains largely

\footnotetext{
${ }^{1}$ For instance, Economides (1995), Katz and Shapiro (1985, 1994), and Takeyama (1994).
} 
the same contents, although often of discounted quality. They do not have the nice physical features that the physical form products have, and the quality of the contents are often inferior. (Typically, CD soundtracks are ripped into mp3 files, and DVD movies are ripped into mpg4 Divx files.)

When analyzing the P2P network, it is natural to consider network externalities. Papers such as Gayer and Shy (2003) and Peitz and Waelbroek (2006) analyze the complementary effect of $\mathrm{P} 2 \mathrm{P}$ on the $\mathrm{CD}$ sales by allowing multi-homing; that is, consumers can download the MP3 files and still buy the CDs. The inclusion of positive consumptive externalities is crucial for this complementary effect to exist. The basic rationale is that when more consumers use a product, legally or illegally, the value of this product increases for all consumers (legal and illegal). Another type of network externalities that universally exist for all files shared on the P2P networks concern the downloading costs. A higher number of users of a network improves the availability of the files and hence decreases the standard search costs and downloading time. In this paper, we emphasize solely on this latter direct network effect, and hence focus, in particular, on the substitutional effect between the network and the store.

We model the competition between information content providers and $\mathrm{P} 2 \mathrm{P}$ networks using a two-stage game model. In the first stage, the firm sets a price for the physical form of the product in the store. Then, in the second stage, after having observed the price set by the firm, consumers simultaneously and independently decide whether to buy the physical form from the store, to download the digital form via the P2P network, or not to acquire the content at all. Notice that we consider the single-homing case only, in order to capture the substitutional effect. The consumers are heterogenous with respect to their taste regarding the physical form and the digital form. In their decision, consumers mutually anticipate each others' decisions, since the resulting network size determines the actual costs of downloading. Moreover, the model contains a parameter called the generic cost factor of downloading which represents a collection of factors that may affect downloading costs, for instance, population computer literacy, the availability of broadband internet infrastructure, and most importantly, the degree of legal enforcement of intellectual property rights.

Note that the digital form of the product is not provided by the firm. Thus, the firm can decide on the price of the physical form, but has no (explicit) power to decide on the use of the digital form. The provider of the digital form (the P2P network) is not a strategic player, since there is no price to charge and no profit to generate. Hence, the price of the physical form is, in our model, the only strategic decision variable from the supply side. 
We find that the market may exhibit three different structures. Firstly, the firm may act as a traditional monopoly, either with a partially- or a fully-served market. In particular, the $\mathrm{P} 2 \mathrm{P}$ network does not form. For this to occur, the intrinsic value of the physical form has to be substantially higher than that of the digital form. Secondly, the firm may deter entry of the network by means of a limit pricing policy. Again, the firm either partially or fully serves the market. Finally, the store and the P2P network may co-exist. In this case, the market is guaranteed to be fully served.

Regarding the effect of the generic cost factor of downloading, we find that the larger this cost factor is, the less likely a P2P network will form, and the higher the firm's profit will be. Strikingly, once the market exhibits co-existence of the two platforms, the smaller the cost factor is, the higher the firm sets its price. Despite this peculiar pricing behavior, the firm's profit unambiguously declines as the cost factor decreases. In the partially-served monopolistic market with limit pricing and the fully-served market with multi-platform co-existence, the total welfare decreases in the cost factor. This implies that it is welfare enhancing if the authorities do not over-emphasize on the protection of the IPR for those contents whose creators are not significantly hurt by consumers' free-riding.

The remainder of the paper is organized as follows. In Section 2 the two-stage model is described in detail. Next, the consumers' choices in the second stage, given the price set by the firm in the first stage, are presented in Section 3. Subsequently, in Section 4, the firm's pricing decision in the first stage, and the resulting market structures, are analyzed. Here, in order to reduce multiplicity of equilibria, we opt for an equilibrium selection that is conventional in the network competition literature. The comparative statics of this equilibrium with respect to the model's parameters are provided in Section 5. Section 6 concludes.

\section{The model}

We adopt a two-stage game model. In stage 1 , the firm sets a price $p$ for the physical form of the product in the store. Next, in stage 2, after having observed the price set by the firm, consumers decide simultaneously and independently whether to purchase the physical form from the store $(S)$, to download the digital form via the P2P network $(N)$, or not to acquire the product at all $(\emptyset)$. We assume that there is a continuum of consumers who differ in their relative preference of the physical form over the digital form. A strategy of the consumer with identity $x \in[0,1]$ is a function $d^{x}$ that maps any price $p$ into her choice set:

$$
d^{x}: \mathbb{R}_{+} \rightarrow\{S, N, \emptyset\}
$$


and we denote the profile of strategies, one for each consumer, by $d=\left(d^{x}\right)_{x \in[0,1]}$.

The price $p$ set by the firm and the profile of consumers' strategies, directly determine the sales from the stores, $s(p, d)$, and the size of the $\mathrm{P} 2 \mathrm{P}$ network, $n(p, d)$, via:

$$
s(p, d)=\mu\left(\left\{x \in[0,1] \mid d^{x}(p)=S\right\}\right) \quad \text { and } \quad n(p, d)=\mu\left(\left\{x \in[0,1] \mid d^{x}(p)=N\right\}\right),
$$

where $\mu$ denotes the Lebesgue measure. ${ }^{2}$

We assume, for simplicity, that the firm has zero costs in production and aims to maximize its profit, given by

$$
\pi(p, d)=p \cdot s(p, d) .
$$

Consumers try to achieve a utility level as high as possible. Let the utility of the consumer identified by $x \in[0,1]$ be given by

$$
U^{x}(p, d)= \begin{cases}\beta-\tau x-p & \text { if } d^{x}(p)=S \\ \gamma-\tau(1-x)-C(n(p, d)) & \text { if } d^{x}(p)=N \\ 0 & \text { if } d^{x}(p)=\emptyset,\end{cases}
$$

where $\beta>0$ and $\gamma>0$ represent the basic utility of the physical and the digital form respectively. The identity $x \in[0,1]$ reflects the consumer's relative preference over the two forms. The consumer with identity $x=0$ has a strong preference for the physical form, whereas the consumer with identity $x=1$ has a strong preference for the digital form. For consumers $x \in(0,1)$, the acquisition of one of the forms generates a disutility that depends on the identity, $x$, and the parameter $\tau>0$. The parameter $\tau$ captures the amount of heterogeneity in consumers' tastes. Finally, $C(n)$ represents the costs of downloading when the resulting network is of size $n \in[0,1]$. A natural shape of this cost function is it to be decreasing in the network size at a diminishing rate. We, therefore, use the following convex decreasing cost function,

$$
C(n)=\sigma \cdot(1-n)^{2},
$$

where $\sigma>0$ represents the generic cost factor of downloading, incorporating a collection of factors that may affect downloading costs, for instance, population computer literacy, the availability of broadband internet infrastructure, and most importantly, the degree of legal enforcement of intellectual property rights. Note that $\sigma$ is identical for every consumer and is independent of the network size.

We pose some assumptions on the relevant parameters.

\footnotetext{
${ }^{2}$ In equilibrium, the sets of consumers buying in the store and going to the network, respectively, will be measurable.
} 
Assumption 1. $\beta>\gamma$.

Personal preferences aside, the objective product quality of the original physical form is higher than that of the digital form (which is "ripped" from the original).

Assumption 2. $\beta>\tau$ and $\gamma>\tau$.

Every consumer prefers a free physical product and a costless download to not acquiring the product.

Assumption 3. $\sigma>\gamma$.

The generic cost factor of downloading is sufficiently high such that when the network size is zero, no consumer can join the network.

Although irrelevant for the economic implications resulting from the model, we impose some assumptions on the consumers' behavior in case of indifference. When a consumer is indifferent between the store and not acquiring the product or between the store and the network, she chooses the store. When she is indifferent between the network and not acquiring the product, she chooses the network. Thus, without loss of generality, we assume a linear order of priority of the store $(S)$ above the network $(N)$, and the network $(N)$ above not acquiring the product $(\emptyset)$.

Finally, we restrict attention to the case where the firm's price is bounded from above by the maximum that a consumer is willing to pay for the product; i.e., $0 \leq p \leq \beta$. If $p>\beta$, all consumers would have negative utility level from buying in the store, hence the firm's sales and profit would be zero. The firm can always do better, in equilibrium, by lowering the price to the interval $[0, \beta]$. Hence, this assumption can be made without any loss of generality and does not impose any restrictions on the results.

In the following sections we present the subgame-perfect Nash equilibria of the model. As usual, we start the analysis with determining the possible Nash equilibria for each of the subgames. That is, we determine the Nash equilibria of the game in the second stage that result from each possible first stage price. Then, we consider the firm's pricing behavior in the first stage and find the subgame-perfect Nash equilibria.

\section{Consumers' choice}

First, we observe that if a consumer with identity $x$ chooses for the store, in equilibrium, all consumers with identity less than $x$ choose for the store. Secondly, if a consumer with 
identity $x$ chooses for the network, in equilibrium, all consumers with identity larger than $x$ choose for the network. This implies that, in equilibrium, only a few number of market structures can arise. The market structures can differ in two dimensions: the degree to which consumers are served and the platforms that are actively used. Regarding the first dimension, the market can be fully served or partially served as is displayed in Figure 1 for a multi-platformed market. ${ }^{3}$ Regarding the second dimension we can have a multi-platformed
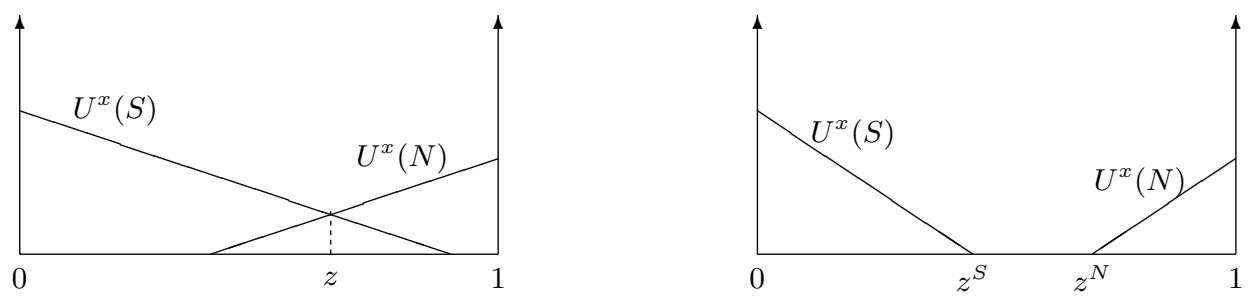

Figure 1: Fully-served market and partially-served market.

market or a single-platformed market with either only the store or only the P2P network. We prove in Proposition 1 that only the six market structures depicted in Table 1 can result in the second stage.

\begin{tabular}{lccc}
\hline & \multicolumn{3}{c}{ single-platformed } \\
& store & network & multi-platformed \\
\hline fully-served & {$[S]$} & {$[N]$} & {$[S / N]$} \\
partially-served & {$[S / \emptyset]$} & {$[\emptyset / N]$} & {$[S / \emptyset / N]$} \\
\hline
\end{tabular}

Table 1: All possible market structures in stage 2.

Observe that, the situation in the graph on the left of Figure 1 is denoted by $[S / N]$; and the one on the right by $[S / \emptyset / N]$.

For the structures involving a P2P network there are two possible equilibria due to network effects. One corresponds to a relatively small network size and the other to a larger one. Along the convention in the network literature, we shall call the former the critical mass network (denoted by c.m. from here on out) and the latter the maximum network (denoted by m.n.). ${ }^{4}$ Furthermore, there is a third possible Nash equilibrium which involves no network at all. Note that we do not make any a priori selections among these equilibria.

For each of the structures we describe the first stage prices that make the structure com-

\footnotetext{
${ }^{3}$ Notice that the vertical intercept of the utility level from the network is endogenously determined by the resulting network size. The quantities $z, z^{S}$ and $z^{N}$ are used for the analysis in the appendices.

${ }^{4}$ See, for instance, Economides and Himmelberg (1995).
} 
patible with a Nash equilibrium of the game in the second stage in the following proposition.

Proposition 1. Given the first stage price p, the possible equilibrium structures that can arise in the second stage are

$$
\begin{array}{ll}
{[S]} & \text { if } 0 \leq p \leq \beta-\tau, \\
{[S / \emptyset]} & \text { if } \beta-\tau<p \leq \beta, \\
{[S / N]_{c . m .}} & \text { if } \beta-\gamma+\tau-\frac{\tau^{2}}{\sigma} \leq p \leq \beta-\frac{\tau^{2}}{2 \sigma}\left(1+\sqrt{1+4 \frac{\sigma}{\tau^{2}}(\gamma-\tau)}\right), \\
{[S / \emptyset / N]_{c . m .} .} & \text { if } \beta-\frac{\tau^{2}}{2 \sigma}\left(1+\sqrt{1+4 \frac{\sigma}{\tau^{2}}(\gamma-\tau)}\right)<p \leq \beta, \\
{[S / N]_{m . n .}} & \text { if } \beta-\gamma+\tau-\frac{\tau^{2}}{\sigma} \leq p \leq \beta-\gamma+\tau, \text { and } \\
{[N]} & \text { if } \beta-\gamma+\tau<p \leq \beta .
\end{array}
$$

Proof.

$[\emptyset]$ and $[\emptyset / N]$. Observe that for these structures to occur, the consumer with identity 0 would get a negative utility from buying in the store, which implies $p>\beta$. This case has been excluded.

$[S]$. Since $\sigma>\gamma$, this structure is consistent with an equilibrium if and only if the consumer with identity 1 is indifferent between not acquiring the product and going to the store, so $\beta-\tau-p \geq 0$, which is the case if and only if $0 \leq p \leq \beta-\tau$.

$[S / \emptyset]$. Since $\sigma>\gamma$, for this structure to occur, the consumer with identity 1 should get a negative utility from buying in the store, which is the case if and only if $p>\beta-\tau$.

$[S / N]$. See Appendix A.

$[S / \emptyset / N]$. See Appendix B.

$[N]$. Note that for all Nash equilibria in this structure $n(p, d)=1$, so the costs of downloading are zero. Since $\gamma>\tau$, this structure appears as an equilibrium if and only if the consumer with identity 0 prefers the network over the store, which is the case if and only if $p>\beta-\gamma+\tau$.

A closer look at Proposition 1 reveals that there are three pairs of logically connected equilibrium structures. They are: (a) the store segment: $[S]-[S / \emptyset]$, (b) the critical-mass network segment: $[S / N]_{c . m .}-[S / \emptyset / N]_{c . m .}$, and (c) the maximum network segment: $[S / N]_{m . n .}-[N]^{.5}$ These three segments share the common property that they begin at a certain price with one of the equilibrium structures, switch to the other equilibrium structure at a higher price and end with that second structure at an even higher price. Table 2 summarizes, for each of the three segments, the starting, switching and ending points. These are denoted by $p_{(\cdot)}^{\vdash}, p_{(\cdot)}^{\times}$and $p_{(\cdot)}^{\dashv}$ respectively.

\footnotetext{
${ }^{5}$ Graphical representations of these segments are presented in Figure 2. Detailed explanations of this figure
} 


\begin{tabular}{llll}
\hline segment & starting price $\left(p_{(\cdot)}^{\vdash}\right)$ & switching price $\left(p_{(\cdot)}^{\times}\right)$ & ending price $\left(p_{(\cdot)}^{-}\right)$ \\
\hline (a) & 0 & $\beta-\tau$ & $\beta$ \\
(b) & $\beta-\gamma+\tau-\frac{\tau^{2}}{\sigma}$ & $\beta-\frac{\tau^{2}}{2 \sigma}\left(1+\sqrt{1+4 \frac{\sigma}{\tau^{2}}(\gamma-\tau)}\right)$ & $\beta$ \\
(c) & $\beta-\gamma+\tau-\frac{\tau^{2}}{\sigma}$ & $\beta-\gamma+\tau$ & $\beta$ \\
\hline
\end{tabular}

Table 2: Three segments of equilibrium structures.

Notice that up to a price of $p_{(b, c)}^{\vdash}=\beta-\gamma+\tau-\frac{\tau^{2}}{\sigma}$ there is a unique equilibrium where all consumers choose the store, and that for higher prices there are three equilibria - one for each segment. At the price $p_{(b, c)}^{\vdash}$ there are two equilibria since the one corresponding to the critical mass network coincides with the maximal network one. It is easily verified that for parameter settings satisfying Assumptions 1-3, for each segment, the switching point is strictly between the starting point and the ending point. Moreover, the switching point of the network segment (b) exceeds the switching point of the store segment (a).

At the store segment (a) only one platform is active, the store. The switching point indicates the price level at which the market switches from being fully served to partially served. At the network segment (b), both platforms co-exist. Here, again, the switching point indicates a switch from a fully-served to a partially-served market. For the network segment (c), the switching point indicates a switch from a multi-platformed to a single-platformed market. Along this whole segment the market is fully served.

\begin{tabular}{lccl}
\hline & $p_{(b, c)}^{\vdash} \leq p_{(a)}^{\times}$ & $p_{(b, c)}^{\leftarrow}>p_{(a)}^{\times}$ \\
\hline$p_{(c)}^{\times} \geq p_{(b)}^{\times}$ & A.1 & B.1 & $\gamma \leq 2 \tau$ and $\left(\gamma \leq \frac{\tau^{2}}{2 \sigma}+\tau\right.$ or $\left.\gamma \geq \frac{2 \tau^{2}}{\sigma}+\tau\right)$ \\
$p_{(c)}^{\times} \in\left[p_{(a)}^{\times}, p_{(b)}^{\times}\right]$ & A.2 & B.2 & $\gamma \leq 2 \tau$ and $\frac{\tau^{2}}{2 \sigma}+\tau \leq \gamma \leq \frac{2 \tau^{2}}{\sigma}+\tau$ \\
$p_{(c)}^{\times} \leq p_{(a)}^{\times}$ & A.3 & - & $\gamma \geq 2 \tau$ \\
\hline \multicolumn{5}{r}{} \\
\hline
\end{tabular}

Table 3: Possible scenarios and their parameter settings.

Different parameters values can lead to various scenarios, depending on how the starting point of the network segments (b) and (c) are related to the switching point of the store segment (a) and how the switching point of network segment (c) is positioned relative to the switching point of the other two segments. Table 3 shows all five possible scenarios - denoted by A.1-3 and B.1-2. Two of these scenarios (A.1 and B.1) are illustrated in Figure 2.

In addition, Table 3 displays the parameter conditions that determine the respective are provided later. 

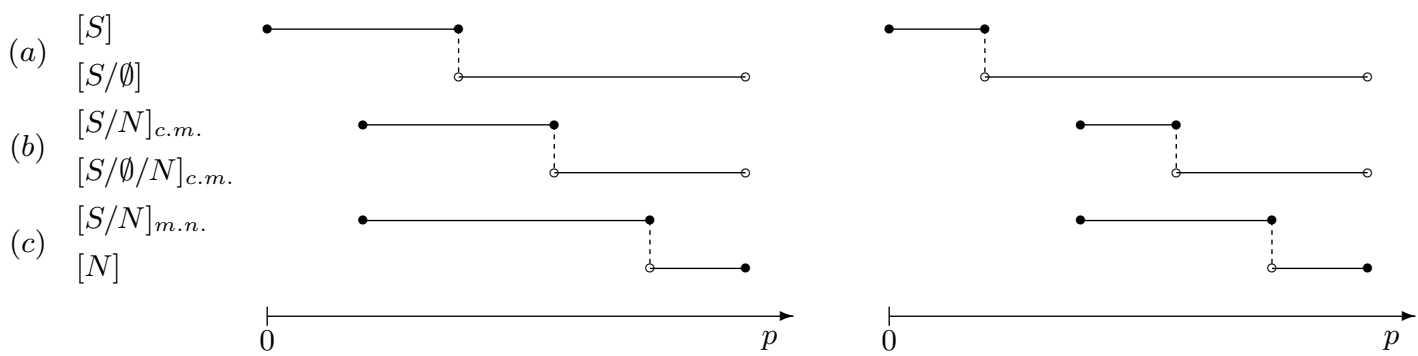

Figure 2: scenario A.1 (left); scenario B.1 (right).

scenarios (last row and last column). We see that the respective scenarios only depend on the parameters $\gamma, \tau$ and $\sigma$, and are independent of $\beta$.

For $\sigma=1$, Figure 3 displays, in the $(\tau, \gamma)$-space, how the scenarios are related to one another. Notice that we can take $\sigma=1$ without loss of generality since all inequalities in

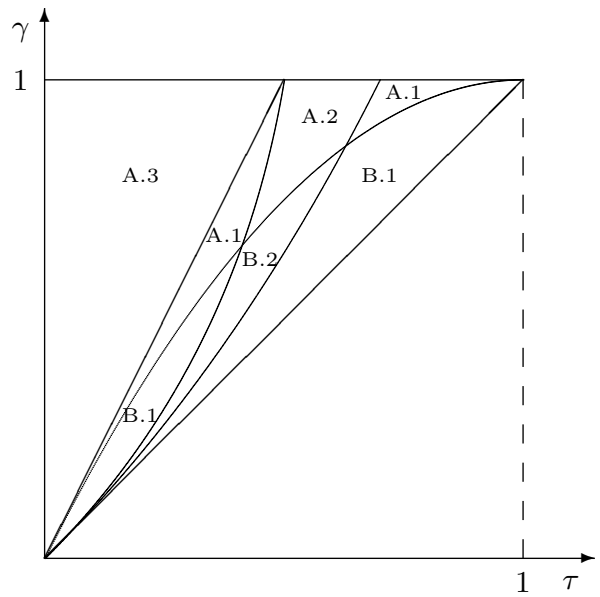

Figure 3: Parameter settings leading to each of the scenarios.

Table 3 are homogeneous. Whenever $(\gamma, \tau, \sigma)$ satisfies the conditions of a particular scenario in Table 3 , so does $\left(\frac{\gamma}{\sigma}, \frac{\tau}{\sigma}, 1\right)$.

In Figure 3, the concave-shaped curve separates the A-scenarios, with the network segments (b) and (c) starting before the store segment (a) switches, from the B-scenarios, with the store segment (a) switching before the network segments (b) and (c) start. Roughly speaking, $\gamma$ and $\tau$ have to be relatively close for B-scenarios to occur, and conversely $\gamma$ has to be sufficiently larger than $\tau$ for A-scenarios to occur. Intuitively, when $\gamma$ is large compared to $\tau$, the desirability of the digital form dominates the transportation costs caused by taste heterogeneity, which makes people located closer to the physical form more prone to 
downloading. This leads to A-scenarios, where the firm perceives a lot of pressure from the network.

Finally, before moving on to the analysis of stage 1, the following proposition specifies (independent of the scenario) the behavior of the firm's profit along each of the segments. Its proof is straightforward and therefore omitted.

Proposition 2. Along each segment the profit of the firm is continuous in the price. For each price, the profit on segment (a) is larger than the profit on segment (b), which is in turn larger than the profit on segment (c). At the ending point the profit is zero on each segment. Along segment (a) the profit is equal to the price until the switching point and is concave afterwards. The profit at the starting point on segment (b) and (c) is equal to $\frac{\tau}{\sigma}$ times the price. Along segment (c) the profit is zero after the switching point.

Intuitively, for a given price with multiple equilibria in the second stage, the firm always has a higher profit if the network is not formed. At the ending point of each segment the price is $\beta$, which will result in no sales, and hence zero profit. Before the switching point on segment (a), the sales quantity is exactly 1 (the full market), therefore the profit is equal to the price. After the switching point, sales decrease in price and the profit is therefore concave in price. At the starting point of segment (b) and (c), the network size is identical for the critical mass network and the maximum network, and sales are less than one. After this starting point, the critical mass network size starts to decrease and the maximum network size starts to increase. The profit along segment (c) after the switching point is zero because there are no sales.

\section{The firm's decision}

\subsection{Subgame-perfect Nash equilibria}

Using the definition of a subgame-perfect Nash equilibrium and the results of the previous section, the following result is immediate.

Proposition 3. The strategy profile $\left(p^{*}, d^{*}\right)$ is a subgame-perfect Nash equilibrium if and only if, for every $p \in[0, \beta], d^{*}(p)$ is consistent with one of the second-stage equilibrium structures of Proposition 1 and $\pi\left(p^{*}, d^{*}\left(p^{*}\right)\right) \geq \pi\left(p, d^{*}(p)\right)$.

In order to analyze which pairs of prices and consumer decisions $(p, d(p))$ can be supported as a subgame-perfect equilibrium outcome, it is convenient to define the firm-worst response of the consumers. Let therefore $d_{\mathrm{LE}}$ be the mapping that assigns to each price the equilibrium 
structure that generates the lowest profit for the firm, except at the starting price $p=$ $\beta-\gamma+\tau-\frac{\tau^{2}}{\sigma}$ of the network segments, where we define $d_{\mathrm{LE}}(p)$ as the store equilibrium structure, so $d_{\mathrm{LE}}^{x}(p)=S, x \in[0,1]$. Here, LE stands for "lower envelope". For the five scenarios depicted in Table 3, the corresponding lower envelope mappings are depicted in Figure 4. Notice that the lower envelope of the three A-scenarios has the structure as displayed
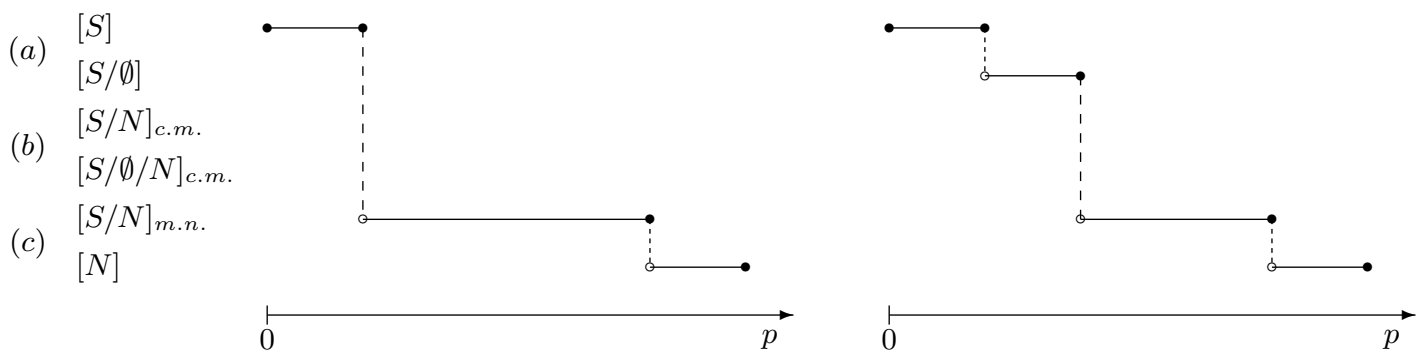

Figure 4: The lower envelope of the different scenarios. left: A-scenarios; right: B-scenarios.

in the left panel and the two B-scenarios as displayed in the right panel. Moreover, the lower envelope only involves segments (a) and (c), but not segment (b). We define the maximum profit along the lower envelope by $\pi_{\mathrm{LE}}^{*}=\max _{p} \pi\left(p, d_{\mathrm{LE}}(p)\right)$. Proposition 2 and the definition of the lower envelope guarantee that the maximum exists, even though $\pi\left(p, d_{\mathrm{LE}}(p)\right)$ is not everywhere continuous in $p$. We have the following characterization of subgame-perfect equilibrium outcomes.

Proposition 4. The pair $\left(p^{*}, d^{*}\left(p^{*}\right)\right)$ is a subgame-perfect Nash equilibrium outcome if and only if $d^{*}\left(p^{*}\right)$ is consistent with one of the second stage equilibrium structures of Proposition 1 and $\pi\left(p^{*}, d^{*}\left(p^{*}\right)\right) \geq \pi_{\mathrm{LE}}^{*}$.

Proof.

$(\Rightarrow)$ Let $\left(p^{*}, d^{*}\right)$ be a subgame-perfect Nash equilibrium with equilibrium outcome $\left(p^{*}, d^{*}\left(p^{*}\right)\right)$. Proposition 3 implies that $d^{*}\left(p^{*}\right)$ is consistent with one of the second stage equilibrium structures of Proposition 1. Since, given $d^{*}$ the firm has no incentive to deviate from $p^{*}$, we have, for every $p \in[0, \beta]$,

$$
\pi\left(p^{*}, d^{*}\left(p^{*}\right)\right) \geq \pi\left(p, d^{*}(p)\right) .
$$

For $p \in[0, \beta] \backslash\left\{\beta-\gamma+\tau-\frac{\tau^{2}}{\sigma}\right\}$, it holds that $\pi\left(p, d^{*}(p)\right) \geq \pi\left(p, d_{\mathrm{LE}}(p)\right)$, so

$$
\begin{aligned}
\pi\left(p^{*}, d\left(p^{*}\right)\right) & \geq \sup _{p \in[0, \beta] \backslash\left\{\beta-\gamma+\tau-\frac{\tau^{2}}{\sigma}\right\}} \pi\left(p, d_{\mathrm{LE}}(p)\right) \\
& =\sup _{p \in[0, \beta]} \pi\left(p, d_{\mathrm{LE}}(p)\right) \\
& =\pi_{\mathrm{LE}}^{*}
\end{aligned}
$$


where the first inequality uses that $\pi\left(p, d_{\mathrm{LE}}(p)\right)$ is continuous from the left at $p=\beta-\gamma+\tau-\frac{\tau^{2}}{\sigma}$. $(\Leftarrow)$ We define the consumers' strategy profile $\widetilde{d}_{\mathrm{LE}}$ by $\widetilde{d}_{\mathrm{LE}}\left(p^{*}\right)=d^{*}\left(p^{*}\right)$ and $\widetilde{d}_{\mathrm{LE}}(p)=d_{\mathrm{LE}}(p)$ for $p \neq p^{*}$. By Proposition $3,\left(p^{*}, \widetilde{d}_{\mathrm{LE}}\right)$ is a subgame-perfect Nash equilibrium, and therefore $\left(p^{*}, d^{*}\left(p^{*}\right)\right)$ a subgame-perfect Nash equilibrium outcome, if $\pi\left(p^{*}, \widetilde{d}_{\mathrm{LE}}\left(p^{*}\right)\right) \geq \pi\left(p, \widetilde{d}_{\mathrm{LE}}(p)\right)$ for every $p \in[0, \beta]$. This inequality holds since, by assumption, $\pi\left(p^{*}, \widetilde{d}_{\mathrm{LE}}\left(p^{*}\right)\right) \geq \pi_{\mathrm{LE}}^{*}$, and $\pi_{\mathrm{LE}}^{*} \geq \pi\left(p, \widetilde{d}_{\mathrm{LE}}(p)\right)$ for $p \neq p^{*}$ by definition of $\pi_{\mathrm{LE}}^{*}$.

\subsection{Equilibrium selection}

To deal with the multiplicity of subgame-perfect equilibria, we follow the convention by supposing that, once the price is known, consumers coordinate on the equilibrium continuation that they prefer, which is the one with the largest network size. ${ }^{6}$ To avoid continuity problems, we assume coordination on the store for the knife-edge case when $p=\beta-\gamma+\tau-\frac{\tau^{2}}{\sigma}$. Incidentally, this corresponds to the firm-worst response of the consumers, which is captured by our lower envelope. In this subsection, we therefore study the firm's optimal pricing behavior along the lower envelope. Inspired by Figure 4, we treat the A-scenarios and B-scenarios separately. With 'scenario A' we refer to all A-scenarios; with 'scenario B' to all B-scenarios. For convenience in notation, we define $\delta \equiv 4 \tau^{2}-3 \sigma(\beta-\gamma+\tau)$. Without loss of generality, we assume that when different first-stage prices lead to identical second-stage profit levels, the firm selects the price that results in the market structure with the smallest possible network size.

Scenario A. In scenario A, the firm's profit along the lower envelope is given by:

$$
\pi\left(p, d_{\mathrm{LE}}(p)\right)= \begin{cases}p & \text { if } 0 \leq p \leq \beta-\gamma+\tau-\frac{\tau^{2}}{\sigma} \\ p \cdot \frac{\tau-\sqrt{\sigma(p+\gamma-\beta-\tau)+\tau^{2}}}{\sigma} & \text { if } \beta-\gamma+\tau-\frac{\tau^{2}}{\sigma}<p \leq \beta-\gamma+\tau \\ 0 & \text { if } \beta-\gamma+\tau<p \leq \beta\end{cases}
$$

The profit on the interval $[N]$ is zero and therefore uninteresting. The profit on the interval $[S]$ reaches its maximum at the right boundary point, which we denote by $\pi_{[S]}^{*}=\beta-\gamma+\tau-\frac{\tau^{2}}{\sigma}$. The profit on the interval $[S / N]_{m . n}$. starts at $\frac{\tau}{\sigma}\left(\beta-\gamma+\tau-\frac{\tau^{2}}{\sigma}\right)$, which is a share $\frac{\tau}{\sigma}$ of $\pi_{[S]}^{*}$. At this point it decreases with a slope of $-\infty$, and ends up equal to zero at the price $p_{(c)}^{\times}=\beta-\gamma+\tau$. However, before it reaches zero, it may increase and then subsequently decrease, achieving a local maximum at the price

$$
p_{[S / N]_{m . n .}}^{*}=\frac{2}{9 \sigma}\left(2 \tau^{2}-\delta+\tau \sqrt{\delta}\right)=\frac{2}{9 \sigma}(2 \tau-\sqrt{\delta})(\tau+\sqrt{\delta}) .
$$

\footnotetext{
${ }^{6}$ See, for instance, Farrell and Saloner (1985), Katz and Shapiro (1986), or Fudenberg and Tirole (2000).
} 
This expression is only valid if $\delta$ is non-negative, which incidentally also guarantees that the price is in the respective interval, i.e. $\delta \geq 0$ implies $p_{(c)}^{\vdash}<p_{[S / N]_{m . n}}^{*}<p_{(c)}^{\times}$. In that case, the profit at this local maximum is

$$
\pi_{[S / N]_{m . n .}}^{*}=\frac{2}{27 \sigma^{2}}(2 \tau-\sqrt{\delta})(\tau+\sqrt{\delta})\left(3 \tau-\sqrt{\tau^{2}+\delta+2 \tau \sqrt{\delta}}\right)=\frac{2}{27 \sigma^{2}}(2 \tau-\sqrt{\delta})^{2}(\tau+\sqrt{\delta}) .
$$

Thus, the firm sets a price of $p_{[S / N]_{m . n} \text {. }}^{*}$ if and only if $\delta \geq 0$ and $\pi_{[S / N]_{m . n .}}^{*}>\pi_{[S]}^{*}$. This leads to a multi-platformed fully-served market with co-existence of store and network. If $\delta \geq 0$, but $\pi_{[S / N]_{m . n .}^{*}}^{*} \leq \pi_{[S]}^{*}$, the firm sets an entry-deterring price equal to $\beta-\gamma+\tau-\frac{\tau^{2}}{\sigma}$, which leads to a single-platformed fully-served market with limit pricing. ${ }^{7}$ The firm sets the same entry-deterring price in case $\delta<0$.

Scenario B. In scenario B, the firm's profit along the lower envelope is given by:

$$
\pi\left(p, d_{\mathrm{LE}}(p)\right)= \begin{cases}p & \text { if } 0 \leq p \leq \beta-\tau \\ p \cdot \frac{\beta-p}{\tau} & \text { if } \beta-\tau<p \leq \beta-\gamma+\tau-\frac{\tau^{2}}{\sigma} \\ p \cdot \frac{\tau-\sqrt{\sigma(p+\gamma-\beta-\tau)+\tau^{2}}}{\sigma} & \text { if } \beta-\gamma+\tau-\frac{\tau^{2}}{\sigma}<p \leq \beta-\gamma+\tau \\ 0 & \text { if } \beta-\gamma+\tau<p \leq \beta\end{cases}
$$

Again, the profit on the interval $[N]$ is zero. On the interval $[S]$, the maximum profit is achieved at the price $p=\beta-\tau$. This is a global maximum on the whole lower envelope if and only if $\beta \geq 2 \tau$. In this case, the firm optimally serves the whole market. Next, on the interval $[S / \emptyset]$, the maximum profit is achieved at the price $p=\frac{1}{2} \beta$. This is the global maximum on the lower envelope if and only if $2\left(\gamma-\tau+\frac{\tau^{2}}{\sigma}\right)<\beta<2 \tau$. In that case, the firm optimally serves the market partially as a monopoly, and there will be no network.

If $\beta \leq 2\left(\gamma-\tau+\frac{\tau^{2}}{\sigma}\right)$, the situation is similar to scenario A. The maximum profit on the interval $[S / \emptyset]$ is achieved at the price $p_{(c)}^{\vdash}=\beta-\gamma+\tau-\frac{\tau^{2}}{\sigma}$, yielding $\pi_{[S / \emptyset]}^{*}=(\beta-\gamma+$ $\left.\tau-\frac{\tau^{2}}{\sigma}\right)\left(\frac{\gamma}{\tau}-1+\frac{\tau}{\sigma}\right)$. Thus, the firm sets the price at $p=\frac{2}{9 \sigma}(2 \tau-\sqrt{\delta})(\tau+\sqrt{\delta})$ if and only if $\delta \geq 0$ (which guarantees $p_{(c)}^{\vdash}<p_{[S / N]_{m . n .}}^{*}<p_{(c)}^{\times}$) and $\pi_{[S / N]_{m . n} .}^{*}>\pi_{[S / \emptyset]}^{*}$. This leads to a multi-platformed fully-served market with the co-existence of the store and the network. Otherwise the firm sets an entry-deterring price equal to $\beta-\gamma+\tau-\frac{\tau^{2}}{\sigma}$, which leads to a single-platformed partially-served market with limit pricing.

Observe that, in scenario B, on top of the limit pricing and co-existence cases like in scenario $\mathrm{A}$, we have two cases of monopoly pricing, under the condition that $\beta$ is sufficiently

\footnotetext{
${ }^{7}$ Our assumption that when different first-stage prices lead to identical second-stage profit levels, the firm selects the price that results in the market structure with the smallest possible network size, implies in this case that the firm selects the limit price rather than the co-existence price when both prices lead to the same profit level.
} 
high when compared to $\tau$. Notice also that the limit pricing in scenario B occurs in a partiallyserved market as opposed to a fully-served market in scenario A.

We summarize the findings from our studies of scenarios above in the following propositions.

Proposition 5. The firm acts as a monopolist if and only if

$$
\gamma<2 \tau-\frac{\tau^{2}}{\sigma} \quad \text { and } \quad \beta>2\left(\gamma-\tau+\frac{\tau^{2}}{\sigma}\right) .
$$

If in addition $\beta \geq 2 \tau$, the market is fully served; otherwise it is partially served.

The first inequality in the proposition ensures that we are in scenario B.

The proposition indicates that when the quality of the physical form is sufficiently high and the quality of the digital form is sufficiently low, the firm can monopolize the market. In case the gross value of the product exceeds the transportation costs by far, the firm is willing to serve the whole market. When this is not the case, the firm does not attempt to sell to the consumers with strong preferences for the digital form. Notice that, here, in its pricing the firm disregards the network. This is not the case in the next proposition.

Proposition 6. There are parameter constellations such that, in equilibrium, the firm applies limit pricing. This is the case with a fully-served market if and only if

$$
\gamma \geq 2 \tau-\frac{\tau^{2}}{\sigma} \quad \text { and } \quad\left(\delta<0 \text { or } \pi_{[S / N]_{m . n .}^{*}}^{*} \leq \pi_{[S]}^{*}\right),
$$

and the case with a partially served market if and only if

$$
\gamma<2 \tau-\frac{\tau^{2}}{\sigma}, \quad \beta \leq 2\left(\gamma-\tau+\frac{\tau^{2}}{\sigma}\right), \quad \text { and } \quad\left(\delta<0 \text { or } \pi_{[S / N]_{m . n .}}^{*} \leq \pi_{[S / \emptyset]}^{*}\right) .
$$

When the digital form is above a certain quality level, or when the physical form is below a certain quality level, the network has sufficient potential to form and the firm is no longer able to apply monopoly pricing. Stated differently, the firm is disciplined by the threat of the network forming. However, when the generic cost factor of downloading, $\sigma$, is sufficiently large (such that $\delta$ is negative), the firm is able to apply limit pricing and deters the network from developing.

Proposition 7. The firm and the network co-exist if and only if the conditions in the previous two propositions are not met. This is the case if and only if

$$
\gamma \geq 2 \tau-\frac{\tau^{2}}{\sigma}, \quad \delta \geq 0, \quad \text { and } \quad \pi_{[S / N]_{m . n .}^{*}}^{*}>\pi_{[S]}^{*}
$$


or

$$
\gamma<2 \tau-\frac{\tau^{2}}{\sigma}, \quad \delta \geq 0, \quad \text { and } \quad \pi_{[S / N]_{m . n .}}^{*}>\pi_{[S / \emptyset]}^{*}
$$

In such a case, the market is fully served.

Proof. It holds that the conditions in Proposition 5 and 6 are not met if and only if

$$
\gamma \geq 2 \tau-\frac{\tau^{2}}{\sigma}, \quad \delta \geq 0, \quad \text { and } \quad \pi_{[S / N]_{m . n .}}^{*}>\pi_{[S]}^{*}
$$

or

$$
\gamma<2 \tau-\frac{\tau^{2}}{\sigma}, \quad \beta \leq 2\left(\gamma-\tau+\frac{\tau^{2}}{\sigma}\right), \quad \delta \geq 0, \quad \text { and } \quad \pi_{[S / N]_{m . n .}}^{*}>\pi_{[S / \emptyset]}^{*} .
$$

Now $\delta \geq 0$ implies

$$
\beta \leq \frac{4 \tau^{2}}{3 \sigma}+\gamma-\tau<\frac{2 \tau^{2}}{\sigma}+2(\gamma-\tau)
$$

so we can omit the condition on $\beta$.

As we will see in the example below, for the conditions for this proposition to hold, the two forms of the product should not be too distinct in quality. Moreover, taste heterogeneity costs should be relatively large such that the products are sufficiently differentiated in order for the firm not to be able to completely extinguish the network. Finally, the generic cost factor of downloading should be sufficiently low, such that the critical mass is easily reached.

We close this section with a graphical illustration of how the different equilibrium market structures are located in the parameter space. For the case with both $\beta$ and $\sigma$ equal to 1 , Figure 5 presents the areas described in the propositions in $(\tau, \gamma)$-space, where 'mon', 'lim' and 'co' refer to monopoly (Proposition 5), limit pricing (Proposition 6) and co-existence (Proposition 7) respectively, and 'fs' and 'ps' refers to a fully-served and partially-served market respectively. The concave curve is identical to the one in Figure 3, and separates scenario A (to the left of the curve) from scenario B (to the right of the curve). Monopoly can only occur in scenario B, either if $\tau \leq \frac{1}{2}$ (fully-served market), or if $\tau>\frac{1}{2}$ and $2\left(\gamma-\tau+\tau^{2}\right)<1$ (partially-served market). This suggests that for the monopoly to occur, $\gamma$ should not be too high when compared to $\beta$, so the quality of the physical form of the product should be sufficiently superior to the one of the digital form. Furthermore, the ratio of $\frac{\gamma}{\tau}$ should not be too high, which means that sufficient taste heterogeneity among consumers is necessary. Co-existence, on the other hand, occurs both in scenario A and B, but only in the far upperright corner. This requires $\tau$ to be sufficiently large, which intuitively suggests that when 


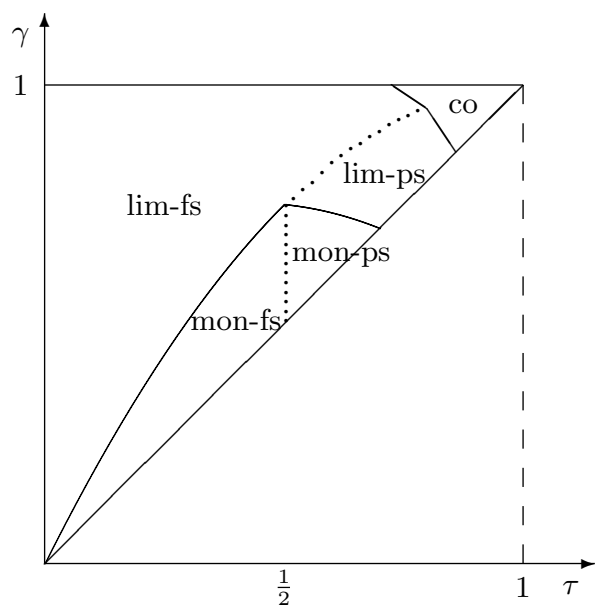

Figure 5: A graphical illustration of the possible subgame-perfect Nash equilibrium market structures.

taste heterogeneity is large, it does not pay off for the firm to deter entry via limit pricing. Hence, the firm will accommodate entry of the network. Everywhere else we find monopoly with limit pricing in either a fully- (in scenario A) or partially-served market (in scenario B). This shows that, in contrast to the popular belief that the content providers are desperately searching for legal help, there is in fact large room for the firm to manipulate the price and deter the entry of the network.

\section{Comparative statics}

In this section, we conduct some comparative statics analysis on the equilibrium outcomes of the subgame-perfect Nash equilibria discussed in the previous section. We will treat them separately according to their market structures. Recall that $p^{*}, s^{*}, n^{*}$, and $\pi^{*}$ are the equilibrium price, sales in the store, network size, and firm's profit respectively. $C S^{*}$ and $W^{*}$ denote equilibrium consumers' surplus and total welfare respectively. Note that due to the absence of production costs, the producer's surplus is equal to the firm's profit.

\subsection{Monopoly}

Proposition 8. The signs of the first order partial derivatives of the equilibrium values with respect to the parameters in the case of the monopoly market are as shown in Table 4. ${ }^{8}$

\footnotetext{
${ }^{8}$ The derivations and proofs of the signs in the tables in Propositions 6, 7 and 8 are shown in Appendix C.
} 


\begin{tabular}{lcccc}
\hline & $\beta$ & $\gamma$ & $\tau$ & $\sigma$ \\
\hline$p^{*}$ & + & 0 & - & 0 \\
$s^{*}$ & 0 & 0 & 0 & 0 \\
$n^{*}$ & 0 & 0 & 0 & 0 \\
$\pi^{*}$ & + & 0 & - & 0 \\
$C S^{*}$ & 0 & 0 & + & 0 \\
$W^{*}$ & + & 0 & - & 0 \\
\hline
\end{tabular}

\begin{tabular}{lcccc}
\hline & $\beta$ & $\gamma$ & $\tau$ & $\sigma$ \\
\hline$p^{*}$ & + & 0 & 0 & 0 \\
$s^{*}$ & + & 0 & - & 0 \\
$n^{*}$ & 0 & 0 & 0 & 0 \\
$\pi^{*}$ & + & 0 & - & 0 \\
$C S^{*}$ & + & 0 & - & 0 \\
$W^{*}$ & + & 0 & - & 0 \\
\hline
\end{tabular}

Table 4: Monopoly pricing with fully-served market (left); Monopoly pricing with partiallyserved market (right). The cells display the signs of the first derivatives.

The findings here are intuitive. For example, in a fully-served monopolistic market, the consumers' surplus does not increase when the basic utility of the physical form of the product, $\beta$, increases, because the price increases by the same amount. The firm's profit increases and hence total welfare increases. An increase in taste heterogeneity, $\tau$, decreases the price by exactly the same amount since the firm must keep the last consumer (whose transportation cost of taste is exactly $\tau$ ) on board. This results in a lower profit for the firm, while consumers benefit from the lower price. In fact, for all but the last consumer the increase in taste heterogeneity is less than the decrease in price. Therefore, the total consumers' surplus increases in taste heterogeneity. Total welfare, however, decreases due to the larger loss in profit. The basic utility of the digital form of the product, $\gamma$, and the generic cost factor of downloading, $\sigma$, do not influence any of the endogenous variables, since small variations in it do not lead to the establishment of the network.

For the partially-served monopoly, a larger basic utility, $\beta$, leads to more sales, higher price, higher profit, and higher welfare, whereas a larger taste heterogeneity, $\tau$, leads to lower sales, lower profit, and lower welfare. Also in the partially-served monopoly, the basic utility of the digital form, $\gamma$, and the generic cost factor of downloading, $\sigma$, do not influence any of the endogenous variables, as in the fully-served monopoly market.

\subsection{Limit pricing}

Proposition 9. The comparative statics on the limit pricing market structure are as shown in Table 5.

In case the market is fully served by the firm, the price is the same as the profit. They both increase in the quality of the physical form, $\beta$, and decrease in that from the 'rival' digital form, $\gamma$. Moreover, they both increase in the generic cost factor of downloading, $\sigma$. As a result, consumers' surplus increases in $\gamma$, thanks to the lower price charged for the product, 


\begin{tabular}{lcccc}
\hline & $\beta$ & $\gamma$ & $\tau$ & $\sigma$ \\
\hline$p^{*}$ & + & - & $+/-$ & + \\
$s^{*}$ & 0 & 0 & 0 & 0 \\
$n^{*}$ & 0 & 0 & 0 & 0 \\
$\pi^{*}$ & + & - & $+/-$ & + \\
$C S^{*}$ & 0 & + & $+/-$ & - \\
$W^{*}$ & + & 0 & - & 0 \\
\hline
\end{tabular}

\begin{tabular}{lcccc}
\hline & $\beta$ & $\gamma$ & $\tau$ & $\sigma$ \\
\hline$p^{*}$ & + & - & $+/-$ & + \\
$s^{*}$ & 0 & + & - & - \\
$n^{*}$ & 0 & 0 & 0 & 0 \\
$\pi^{*}$ & + & - & - & + \\
$C S^{*}$ & 0 & + & $+/-$ & - \\
$W^{*}$ & + & + & - & - \\
\hline
\end{tabular}

Table 5: Fully-served market (left); Partially-served market (right). The cells display the signs of the first derivatives.

but decreases in $\sigma$, due to the higher price. Total welfare increases in $\beta$, thanks to the higher profit, but does not depend on $\gamma$ or $\sigma$. The reason is that in the fully-served market with limit pricing, $\gamma$ and $\sigma$ only have an effect via the price of the product, and therefore only affect the distribution of surplus over the consumers and the firm. An increase in taste heterogeneity, $\tau$, makes consumers less likely to switch platforms, and may lead either to an increase or a decrease in the price. When $\sigma$ is relatively low, in particular if $\sigma<2 \tau$, an increase in $\tau$ leads to a lower price, and consequently lower profits. The consumers' surplus decreases only when $\sigma>\frac{4}{3} \tau$. However, total welfare suffers from higher $\tau$.

A difference we notice when the market is only partially served is that the sales in the store, $s^{*}$, with respect to $\gamma$ and $\sigma$ changes. A decrease in $\gamma$ and an increase in $\sigma$ makes the network less competitive, and triggers an increase in the price, $p^{*}$, and leads then to less sales, $s^{*}$. When it comes to the profit, $\pi^{*}$, the price effect dominates the quantity effect. Thus comparative statics of prices and profits go in the same direction, similar to the case where the market is fully served. Total welfare, $W^{*}$, depends on $\gamma$ and $\sigma$ as well. It goes the opposite direction as the firm's price, $p^{*}$. With a lower $\gamma$ or a higher $\sigma$, leading to a higher price, the gain in profit in the partially-served market is less than that in the fully-served market, due to the lower quantity sold. The effect on the consumers' surplus being the same, total welfare decreases, which explains the positive sign for $\gamma$ and the negative one for $\sigma$.

Even when the network does not form, its attractiveness (represented by a high $\gamma$ and a low $\sigma$ ) does have a negative impact on the firm's profit and a positive impact on the total welfare. This has some implications for intellectual property rights policy. We will discuss this later.

An increase in $\tau$ also affects sales negatively, but the consequences for the price are again ambiguous. As with the fully-served market, the price decreases if $\sigma<2 \tau$ and increases if $\sigma>$ $2 \tau$. Both inequalities on parameter values are consistent with the conditions of Proposition 6 . 
An increase in $\tau$ may now lead to either a higher or a lower consumer surplus, depending on the quantitative significance of the decrease in sales, the increase in transportation costs, and the change in prices. The change in total welfare is unambiguously negative when $\tau$ increases.

\subsection{Co-existence}

Proposition 10. The comparative statics of the co-existence of the store and the network in a fully-served market are as shown in Table 6 .

\begin{tabular}{lcccc}
\hline & $\beta$ & $\gamma$ & $\tau$ & $\sigma$ \\
\hline$p^{*}$ & + & - & + & - \\
$s^{*}$ & + & - & - & + \\
$n^{*}$ & - & + & + & - \\
$\pi^{*}$ & + & - & + & + \\
$C S^{*}$ & - & + & - & - \\
$W^{*}$ & + & + & - & - \\
\hline
\end{tabular}

Table 6: Co-existence of the store and the network with a fully-served market. The cells display the signs of the first derivatives.

Nowadays the co-existence case is often observed in reality, probably most prominently in the music industry. Here we find some very interesting results. An increase in $\beta$ leads to higher prices, sales, and profits, and a lower network size. Surprisingly, consumer surplus is affected negatively by an increase in $\beta$. The negative effects of a higher price and a lower network size outweigh the increase in quality, $\beta$. Total welfare varies positively with $\beta$.

The equilibrium price, $p^{*}$, decreases in the generic cost factor of downloading, $\sigma$, despite the fact that the equilibrium network size, $n^{*}$, decreases in $\sigma$. This implies that, in equilibrium, the higher the cost factor, $\sigma$, the lower the firm sets its price. This is counter-intuitive since a higher $\sigma$ makes the network less strong as a competitor of the firm. As it turns out, this negative correlation is caused by the functional form of the downloading costs, $C(n)$, which is convex and decreasing in the network size, $n$. In other words, since downloading costs decrease with the network size at a diminishing rate, it makes the first few consumers who join the network more vital in determining the costs (or the price, effectively) of downloading. The firm, therefore, has an incentive to "play tough" by means of a low price when the equilibrium network size is relatively small (large $\sigma$ ). Conversely, if $\sigma$ is small, the equilibrium network size is large, and the network is stronger and forms more easily. In that case, the firm can do better by backing off from the competition and charging a high price to reap the most profit out of the customers that are more eager to buy the physical form of the product. This can be seen 
by the equilibrium profit, $\pi^{*}$, being increasing in $\sigma$ despite of the decreasing price. What we observe here, therefore, is a very interesting form of platform competition. Moreover, similar to the partially-served market limit pricing case, total welfare, $W^{*}$, decreases in $\sigma$. This implies that it is welfare enhancing if the internet infrastructure of the country is improved, and legal enforcements of intellectual property rights are relaxed.

The comparative statics with respect to $\tau$ conform to the usual intuition. An increase in $\tau$ leads to higher prices and profits, lower sales, and a bigger network. Consumer surplus and total welfare is affected negatively.

\subsection{Comparative statics across market structures}

In the above subsections we have shown comparative statics in the three different market structures separately. In this subsection we analyze how equilibrium values change across market structures as a function of the most important parameter in the model, $\sigma$. We show graphically how equilibrium price, profit and total welfare change in relation to $\sigma$. In order to make a clear sketch, we display the limit case where $\beta, \gamma$ and $\tau$ are equal to 1 . We allow $\sigma$ to vary on the horizontal axis and put the equilibrium values on the vertical axis. Figure 6 shows the equilibrium price, profit and welfare responding to changes in $\sigma$.
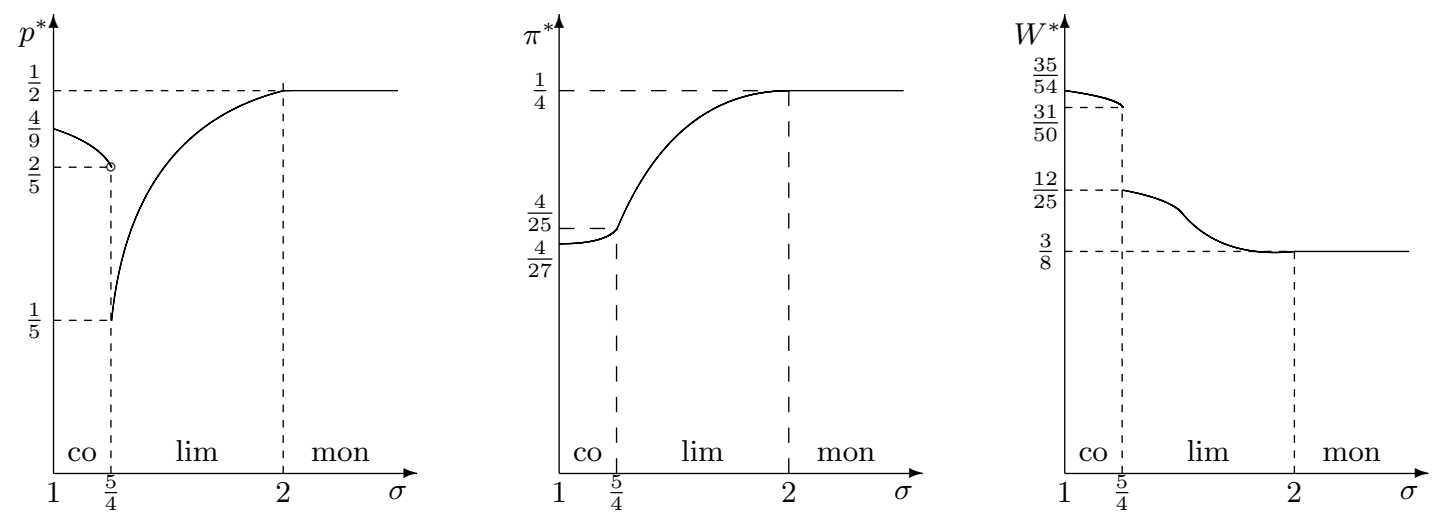

Figure 6: Equilibrium price (left), profit (middle), and welfare (right) across markets structures.

When the generic cost factor of downloading, $\sigma$, is very high, the traditional monopoly setting results, as one would expect. As this cost factor decreases, the price, profit and total welfare remain unaffected as long as the market structure remains unchanged. Once $\sigma$ becomes sufficiently small (less than 2), the firm has to adapt its pricing strategy, because the network becomes a vital enemy to the firm. As long as $\sigma$ is not too small (larger than $\frac{5}{4}$ ), the firm optimally deters entry of the network. In this region, prices and profits are decreasing for 
decreasing values of $\sigma$. Profits are decreasing at an increasing rate. Total welfare, however, increases. In case the cost factor becomes very small $\left(\sigma<\frac{5}{4}\right)$, the firm optimally tolerates the network and refrains from further deterring behavior. This results in a discontinuous increase in price and welfare as the market tips from a single-platformed structure towards a multi-platformed one. From here, the equilibrium price and welfare gradually increase as $\sigma$ decreases. Profits continue to decrease, but only decrease at a diminishing rate.

We conclude that, although society unambiguously benefits from a decrease in the generic cost factor of downloading, the firm unambiguously suffers from it despite a non-monotonic optimal pricing policy. One can, therefore, only expect every attempt possible from the firm to make file-sharing and downloading very difficult and costly indeed.

\section{Concluding remarks}

This paper investigates the competition between an information content provider offering the physical form of the product and a P2P file-sharing network (offering the digital form), where consumers that opt for the P2P network benefit from the presence of other consumers due to decreased search costs and downloading time and increased availability. The model has four parameters that capture the quality of the physical form of the product, the quality of the digital form, the level of consumers' taste heterogeneity, and the generic cost factor of downloading. This latter factor incorporates elements such as population computer literacy, the availability of broadband internet infrastructure, and most importantly, the degree of legal enforcement of intellectual property rights.

We give a complete characterization of the subgame-perfect Nash equilibria of the model as well as of the subgame-perfect Nash equilibrium outcomes. We show that, depending on the parameter values, several market structures may result. These market structures differ not only in the number of platforms being active (one or two) and the level at which the market is served (fully or partially), but also the way these market structures are achieved. A market structure without network is compatible both with monopoly pricing by the firm as well as with an entry-deterring pricing policy. Given fixed values for the other parameters, as we gradually decrease the generic cost factor of downloading, the market experiences first the single platform (the store) with monopoly pricing, then the single platform (the store) with limit pricing, and finally co-existence of multiple competing platforms (the store and the network).

In addition, we find that the equilibrium price moves in the opposite direction of the 
generic cost factor of downloading in case both platforms co-exist. This may seem counterintuitive, since a higher cost factor of downloading makes the network a less attractive choice to the consumers. However, since downloading costs decrease with the network size at a diminishing rate, it makes the first few consumers who join the network more vital in determining the costs of downloading. The firm, therefore, has an incentive to "play tough" by means of a low price when the equilibrium network size is relatively small, which is the case with a relatively high generic cost factor of downloading. Conversely, if the generic cost factor of downloading is relatively low, the equilibrium network size will be large, and the network will form more easily. In that case, the firm can do better by backing off from the competition and charging a high price to reap the most profit out of the customers that are more eager to buy the physical form. Although, in general, the price behaves non-monotonically when the generic cost factor of downloading becomes smaller, profits unambiguously decrease. Total welfare, on the other hand, unambiguously increases. Bearing in mind that we have ignored the existing consumptive externalities that are associated with file-sharing-which are shown in the literature to affect firm's profit and welfare positively - as well as the possibility for multi-homing, the positive impact of file-sharing on social welfare can only be underestimated here.

Based on the results of this paper, one may come to certain conclusions regarding policies towards intellectual property rights protections. One of them is that the involved authorities should be careful in overemphasizing the protection of IPR, provided that contents creators are not significantly hurt by free-riding from the consumers' side.

The music industry is a prime example where this applies. It is sometimes easy to confuse recording companies and musicians, the latter being the content creator, and the former not. ${ }^{9}$ Music downloaders constitute an enormous fan club, which leads to future potential profits in the form of either concert performances, commercial promotions, or even CD sales. Not only are the musicians not hurt by the downloaders, they may in fact benefit from them. Indeed, when was the last time one saw a popular musician short of money because his/her music was downloaded through the internet by millions of people? The real losers in this case are the recording companies who used to enjoy rents from both the musicians and the consumers. They are now faced with increased competition from independent musicians who make themselves famous through the internet.

\footnotetext{
${ }^{9}$ Gayer and Shy (2006) focuses on the conflict in interests between these two parties.
} 


\section{References}

[1] Blackburn D (2004). On-line piracy and recorded music sales. Working Paper: Harvard University.

[2] Economides N (1996). The economics of networks. International Journal of Industrial Organization 14: 673-699.

[3] Economides N and C Himmelberg (1995). Critical mass and network evolution in telecommunications. In: G Brock (ed.) Toward a Competitive Telecommunications Industry: Selected Papers from the 1994 Telecommunications Policy Research Conference.

[4] Farrell J and G Saloner (1985). Standardization, compatibility, and innovation. Rand Journal of Economics 16: 70-83.

[5] Fudenberg D and J Tirole (2000). Pricing a network good to deter entry. The Journal of Industrial Economics 48: 373-390.

[6] Gayer A and O Shy (2003). Internet and peer-to-peer distributions in markets for digital products. Economics Letters 81: 197-203.

[7] Gayer A and O Shy (2006). Publishers, artists, and copyright enforcement. Information Economics \&3 Policy 18: 374-384.

[8] Katz M and C Shapiro (1985). Network externalities, competition and compatibility. The American Economic Review 75: 424-440.

[9] Katz M and C Shapiro (1986). Technology adoption in the presence of network externalities. Journal of Political Economy 94: 822-841.

[10] Katz M and C Shapiro (1994). System competition and network effects. Journal of Economic Perspectives 2: 93-115.

[11] Liebowitz S (2004). Will MP3 downloads annihilate the record industry? The evidence so far. Advances in the Study of Entrepeneurship, Innovation, and Economic Growth 15: $229-260$.

[12] Liebowitz S (2006). File sharing: Creative estruction or just plain destruction? Journal of Law and Economics 49: 1-28. 
[13] Oberholzer-Gee F and K Strumpf (2007). The effect of file sharing on record sales: An empirical analysis. Journal of Political Economy 115: 1-42.

[14] Peitz M and P Waelbroeck (2004). The effect of internet piracy on CD sales: Crosssection evidence. Review of Economic Research on Copyright Issues 1: 71-79.

[15] Peitz M and P Waelbroeck (2006). Why the music industry may gain from free downloading - The role of sampling. International Journal of Industrial Organization 24: 907-913.

[16] Rob R and J Waldfogel (2006). Piracy on the high C's: Music downloading, sales displacement, and social welfare in a sample of college students. Journal of Law and Economics 49: 29-62.

[17] Shapiro C and H Varian (1999). Information Rules: A Strategic Guide to the Network Economy. Boston: Harvard Business School Press.

[18] Shy O and J-F Thisse (1999). A strategic approach to software protection. Journal of Economics and Management Strategy 8: 163-190.

[19] Shy O (2001). The Economics of Network Industries. Cambridge University Press, Cambridge Mass.

[20] Takeyama L (1994). The welfare implications of unauthorized reproduction of intellectual property in the presence of network externalities. Journal of Industrial Economcis 42: $155-166$.

[21] Zentner A (2006). Measuring the effect of file sharing on music purchases. Journal of Law and Economics 49: 63-90.

\section{A The fully-served multi-platformed market}

The consumer $z \in(0,1)$ that separates the consumers choosing for the store from those choosing for the network, is herself indifferent between the two options. Moreover this consumer should weakly prefer the store to the option not to acquire the product. So, for a second stage equilibrium $(p, d)$ to generate the structure $[S / N]$, it should hold that

$$
0 \leq U^{z}\left(p, d^{-z}, S\right)=U^{z}\left(p, d^{-z}, N\right) \quad \Longleftrightarrow \quad 0 \leq \beta-\tau z-p=\gamma-\tau(1-z)-\sigma(1-n(p, d))^{2},
$$


where $n(p, d)=1-z$, since the market is fully-served. Solving the inequality for $z$ gives us two solutions:

$$
z_{c . m .}(p)=\frac{\tau+\sqrt{\sigma(p+\gamma-\beta-\tau)+\tau^{2}}}{\sigma} \quad \text { and } \quad z_{m . n .}(p)=\frac{\tau-\sqrt{\sigma(p+\gamma-\beta-\tau)+\tau^{2}}}{\sigma}
$$

where in accordance with network economics terminology, we call the solution corresponding to the smaller network size $\left(z_{c . m}\right)$ the critical mass, and the solution corresponding to the larger network size $\left(z_{m . n .}\right)$ the maximum network. In order to guarantee the solution to be real, we need

$$
p \geq \beta-\gamma+\tau-\frac{\tau^{2}}{\sigma}
$$

For the market structure $[S / N]_{*}$ to exist, the other conditions needed are:

$$
0 \leq z_{*}(p)<1 \quad \text { and } \quad \beta-\tau z_{*}(p)-p \geq 0 .
$$

The next two subsections deal with the maximum network and the critical mass network, respectively.

\section{A.1 The maximum network}

Assume Inequality (1) holds. The maximum network solution exists for the multi-platformed and fully-served market if and only if the following two conditions are satisfied

$$
0 \leq \frac{\tau-\sqrt{\sigma(p+\gamma-\beta-\tau)+\tau^{2}}}{\sigma}<1 \quad \text { and } \quad \beta-\tau \frac{\tau-\sqrt{\sigma(p+\gamma-\beta-\tau)+\tau^{2}}}{\sigma}-p \geq 0 .
$$

The first condition is equivalent to

$$
p \leq \beta-\gamma+\tau \quad \text { and } \quad \sqrt{\sigma(p+\gamma-\beta-\tau)+\tau^{2}}>\tau-\sigma,
$$

where the second inequality is trivially satisfied owing to the assumption that $\sigma>\tau$ and using Inequality (1). The second condition is equivalent to

$$
\sqrt{\sigma(p+\gamma-\beta-\tau)+\tau^{2}} \geq \tau-\frac{\sigma}{\tau}(\beta-p)
$$

We will argue next that the second condition is always satisfied. In case the right-hand side of the inequality above is negative, i.e. in case $p<\beta-\frac{\tau^{2}}{\sigma}$, the inequality is trivially satisfied. Otherwise, $p \geq \beta-\frac{\tau^{2}}{\sigma}$, and the inequality above implies

$$
\frac{\sigma}{\tau^{2}}(\beta-p)^{2}-(\beta-p)-(\gamma-\tau) \leq 0,
$$


which is equivalent to

$$
\beta-\frac{\tau^{2}}{2 \sigma}\left(1+\sqrt{1+\frac{4 \sigma}{\tau^{2}}(\gamma-\tau)}\right) \leq p \leq \beta-\frac{\tau^{2}}{2 \sigma}\left(1-\sqrt{1+\frac{4 \sigma}{\tau^{2}}(\gamma-\tau)}\right) .
$$

Because $\gamma>\tau$, the lower bound on $p$ is less than $\beta-\frac{\tau^{2}}{\sigma}$ and therefore satisfied since we are considering the case $p \geq \beta-\frac{\tau^{2}}{\sigma}$. The upper bound is larger than $\beta$. Hence the second condition does not impose a further restriction on $p$.

Hence, Inequalities (1) and (2) boil down to

$$
\beta-\gamma+\tau-\frac{\tau^{2}}{\sigma} \leq p \leq \beta-\gamma+\tau
$$

\section{A.2 The critical mass network}

Assume Inequality (1) holds. The critical mass network solution exists for the multi-platformed and fully-served market if and only if the following two conditions are satisfied

$$
0 \leq \frac{\tau+\sqrt{\sigma(p+\gamma-\beta-\tau)+\tau^{2}}}{\sigma}<1 \quad \text { and } \quad \beta-\tau \frac{\tau+\sqrt{\sigma(p+\gamma-\beta-\tau)+\tau^{2}}}{\sigma}-p \geq 0,
$$

Notice that the first inequality of the first condition is trivially satisfied and that the second inequality of this condition can be simplified to

$$
p<\beta-\gamma-\tau+\sigma \text {. }
$$

The second condition is equivalent to

$$
\sqrt{\sigma(p+\gamma-\beta-\tau)+\tau^{2}} \leq \frac{\sigma}{\tau}(\beta-p)-\tau
$$

This condition holds if and only if its right-hand side of this inequality is non-negative - that is $p \leq \beta-\frac{\tau^{2}}{\sigma}$-and

$$
\frac{\sigma}{\tau^{2}}(\beta-p)^{2}-(\beta-p)-(\gamma-\tau) \geq 0
$$

where the latter inequality is equivalent to

$$
p \leq \beta-\frac{\tau^{2}}{2 \sigma}\left(1+\sqrt{1+\frac{4 \sigma}{\tau^{2}}(\gamma-\tau)}\right) \quad \text { or } \quad p \geq \beta-\frac{\tau^{2}}{2 \sigma}\left(1-\sqrt{1+\frac{4 \sigma}{\tau^{2}}(\gamma-\tau)}\right) .
$$

Since $\gamma>\tau$, the right-hand side of the second inequality exceeds $\beta$ and hence cannot be satisfied - leaving the first inequality to be satisfied. Moreover, the right-hand side of the first inequality is less than $\beta-\frac{\tau^{2}}{\sigma}$, such that the second condition is satisfied if and only if

$$
p \leq \beta-\frac{\tau^{2}}{2 \sigma}\left(1+\sqrt{1+\frac{4 \sigma}{\tau^{2}}(\gamma-\tau)}\right) .
$$


Next we show that the second condition is more restrictive than the first condition. Suppose the opposite holds true. That is, suppose that

$$
\beta-\frac{\tau^{2}}{2 \sigma}\left(1+\sqrt{1+\frac{4 \sigma}{\tau^{2}}(\gamma-\tau)}\right)>\beta-\gamma-\tau+\sigma .
$$

We will derive a contradiction. The supposition implies

$$
\sqrt{1+\frac{4 \sigma}{\tau^{2}}(\gamma-\tau)}<\frac{2 \sigma}{\tau^{2}}(\gamma+\tau-\sigma)-1
$$

For Inequality (4) to be satisfied, the right-hand side has to be positive, which is the case when

$$
2 \sigma(\gamma+\tau-\sigma)-\tau^{2}>0
$$

Inequality (4) then implies that

$$
\frac{\sigma}{\tau^{2}}(\gamma+\tau-\sigma)^{2}>2 \gamma-\sigma
$$

which in turn implies that

$$
2 \tau^{2}+\sigma(\sigma-\gamma)-2 \tau \sigma>0
$$

We argue that Inequality (5) and (6) cannot be satisfied simultaneously. By adding Inequality (5) to twice Inequality (6) we find that $\tau>\frac{2}{3} \sigma$. Since we deal with two homogenous polynomial inequalities, we can set one of the parameters to any positive number. We set $\gamma=1$ and realize that this induces $\tau<1<\sigma$. Moreover, $\tau>\frac{2}{3} \sigma$ implies that $\sigma<\frac{3}{2}$. If we solve Inequality (6) for $\tau$, we find

$$
\tau<\frac{\sigma-\sqrt{2 \sigma-\sigma^{2}}}{2} \quad \text { or } \quad \tau>\frac{\sigma+\sqrt{2 \sigma-\sigma^{2}}}{2} .
$$

The first of these inequalities is in direct conflict with $\tau>\frac{2}{3} \sigma$, which leaves the second inequality as the only feasible option. However, for this inequality to hold true, $\frac{\sigma+\sqrt{2 \sigma-\sigma^{2}}}{2}$ has to be less than 1 , so $\sigma$ has to be less than 1 or to be larger than 2 , which contradicts $\sigma \in\left(1, \frac{3}{2}\right)$. We have obtained a contradiction to the supposition. Hence,

$$
\beta-\frac{\tau^{2}}{2 \sigma}\left(1+\sqrt{1+\frac{4 \sigma}{\tau^{2}}(\gamma-\tau)}\right) \leq \beta-\gamma-\tau+\sigma .
$$

Hence, Inequalities (1) and (3) boil down to

$$
\beta-\gamma+\tau-\frac{\tau^{2}}{\sigma} \leq p \leq \beta-\frac{\tau^{2}}{2 \sigma}\left(1+\sqrt{1+\frac{4 \sigma}{\tau^{2}}(\gamma-\tau)}\right) .
$$




\section{B The partially-served multi-platformed market}

The consumer $z^{S} \in(0,1)\left(z^{N} \in(0,1)\right)$ that separates the consumers choosing for the store (network) from those choosing not to acquire any product, is herself indifferent between the two options. Moreover, this consumer prefers as well the store (network) as no acquisition to the option to acquire via the network (store). We have a partially-served market if there is an interval of consumers that prefers no acquisition to acquisition, meaning that $z^{S}$ is less than $z^{N}$. So, for a second stage equilibrium $(p, d)$ to generate the structure $[S / \emptyset / N]$, it should hold that

$$
U^{z^{S}}\left(p, d^{-z^{S}}, S\right)=U^{z^{S}}\left(p, d^{-z^{S}}, \emptyset\right) \text { and } U^{z^{N}}\left(p, d^{-z^{N}}, N\right)=U^{z^{N}}\left(p, d^{-z^{N}}, \emptyset\right)
$$

and

$$
0 \leq z^{S}(p)<z^{N}(p) \leq 1
$$

The first two equations are equivalent to

$$
\beta-\tau z^{S}-p=0 \quad \text { and } \quad \gamma-\tau\left(1-z^{N}\right)-\sigma(1-n(p, d))^{2}=0 .
$$

where $n(p, d)=1-z^{N}$. Solving the first equation for $z^{S}$ and the latter for $z^{N}$ gives us one solution for $z^{S}$ :

$$
z^{S}(p)=\frac{\beta-p}{\tau},
$$

and two solutions for $z^{N}$ :

$$
z_{c . m .}^{N}=\frac{\tau+\sqrt{\tau^{2}+4 \sigma(\gamma-\tau)}}{2 \sigma} \quad \text { and } \quad z_{m . n .}^{N}=\frac{\tau-\sqrt{\tau^{2}+4 \sigma(\gamma-\tau)}}{2 \sigma} .
$$

\section{B.1 The maximum network}

It is easily seen that $z_{m . n}^{N}$. is negative, so the maximum network solution cannot co-exist with a partially-served multi-platformed market.

\section{B.2 The critical mass network}

The critical mass network solution exists for the multi-platformed, partially-served market if and only if

$$
0 \leq \frac{\beta-p}{\tau}<\frac{\tau+\sqrt{\tau^{2}+4 \sigma(\gamma-\tau)}}{2 \sigma} \leq 1 .
$$


The first inequality is satisfied, since $p \leq \beta$. Owing to the assumption that $\sigma>\gamma$, the third inequality is satisfied too. Indeed,

$$
\frac{\tau+\sqrt{\tau^{2}+4 \sigma(\gamma-\tau)}}{2 \sigma}<1 \quad \Longleftrightarrow \quad \sqrt{\tau^{2}+4 \sigma(\gamma-\tau)}<2 \sigma-\tau \quad \Longleftrightarrow \quad \gamma<\sigma .
$$

The second inequality is equivalent to

$$
p>\beta-\frac{\tau^{2}}{2 \sigma}\left(1+\sqrt{1+\frac{4 \sigma}{\tau^{2}}(\gamma-\tau)}\right) .
$$

Hence the conditions in Inequality (7) are satisfied if and only if

$$
\beta-\frac{\tau^{2}}{2 \sigma}\left(1+\sqrt{1+\frac{4 \sigma}{\tau^{2}}(\gamma-\tau)}\right)<p .
$$

\section{Support for Proposition 6-8}

In this Appendix we support our comparative statics results in Section 5. For each of the five different situations we provide the respective equilibrium values. Once a sign of the derivative of these values with respect to one of the model's parameters is not straight-forward (that is, if the factor appears in multiple terms in possibly opposite directions), we provide the precise derivations.

\section{C.1 Monopoly pricing \& fully-served market}

\begin{tabular}{llllll}
\hline & & $\beta$ & $\gamma$ & $\tau$ & $\sigma$ \\
\hline$p^{*}$ & $=\beta-\tau$ & + & 0 & - & 0 \\
$s^{*}$ & $=1$ & 0 & 0 & 0 & 0 \\
$n^{*}$ & $=0$ & 0 & 0 & 0 & 0 \\
$\pi^{*}$ & $=\beta-\tau$ & + & 0 & - & 0 \\
$C S^{*}$ & $=\frac{1}{2} \tau$ & 0 & 0 & + & 0 \\
$W^{*}$ & $=\beta-\frac{1}{2} \tau$ & + & 0 & - & 0 \\
\hline
\end{tabular}

\section{C.2 Monopoly pricing \& partially-served market}

\begin{tabular}{llllll}
\hline & & $\beta$ & $\gamma$ & $\tau$ & $\sigma$ \\
\hline$p^{*}$ & $=\frac{1}{2} \beta$ & + & 0 & 0 & 0 \\
$s^{*}$ & $=\frac{1}{2 \tau} \beta$ & + & 0 & - & 0 \\
$n^{*}$ & $=0$ & 0 & 0 & 0 & 0 \\
$\pi^{*}$ & $=\frac{1}{4 \tau} \beta^{2}$ & + & 0 & - & 0 \\
$C S^{*}$ & $=\frac{1}{8 \tau} \beta^{2}$ & + & 0 & - & 0 \\
$W^{*}$ & $=\frac{3}{8 \tau} \beta^{2}$ & + & 0 & - & 0 \\
\hline
\end{tabular}




\section{C.3 Limit pricing \& fully-served market}

\begin{tabular}{lllccc}
\hline & & $\beta$ & $\gamma$ & $\tau$ & $\sigma$ \\
\hline$p^{*}$ & $=\beta-\gamma+\tau-\frac{\tau^{2}}{\sigma}$ & + & - & $+/-^{1}$ & + \\
$s^{*}$ & $=1$ & 0 & 0 & 0 & 0 \\
$n^{*}$ & $=0$ & 0 & 0 & 0 & 0 \\
$\pi^{*}$ & $=\beta-\gamma+\tau-\frac{\tau^{2}}{\sigma}$ & + & - & $+/-^{1}$ & + \\
$C S^{*}$ & $=\gamma-\frac{3}{2} \tau+\frac{\tau^{2}}{\sigma}$ & 0 & + & $+/-^{2}$ & - \\
$W^{*}$ & $=\beta-\frac{1}{2} \tau$ & + & 0 & - & 0 \\
\hline
\end{tabular}

1. $\operatorname{sign}\left(\frac{\partial p^{*}}{\partial \tau}\right)=\operatorname{sign}\left(\frac{\partial \pi^{*}}{\partial \tau}\right)=\operatorname{sign}\left(1-\frac{2 \tau}{\sigma}\right)=\operatorname{sign}(\sigma-2 \tau)$. We have found parameter values compatible with the conditions in Proposition 6 that support a positive sign as well as a negative one.

2. $\operatorname{sign}\left(\frac{\partial C S^{*}}{\partial \tau}\right)=\operatorname{sign}\left(-\frac{3}{2}+\frac{2 \tau}{\sigma}\right)=\operatorname{sign}\left(\frac{4}{3} \tau-\sigma\right)$. As in 1., both positive and negative signs are found.

\section{C.4 Limit pricing \& partially-served market}

\begin{tabular}{llllcc}
\hline & & $\beta$ & $\gamma$ & $\tau$ & $\sigma$ \\
\hline$p^{*}$ & $=\beta-\gamma+\tau-\frac{\tau^{2}}{\sigma}$ & + & - & $+/-^{1}$ & + \\
$s^{*}$ & $=\frac{1}{\tau}\left(\gamma-\tau+\frac{\tau^{2}}{\sigma}\right)$ & 0 & + & $-{ }^{2}$ & - \\
$n^{*}$ & $=0$ & 0 & 0 & 0 & 0 \\
$\pi^{*}$ & $=\left(\beta-\gamma+\tau-\frac{\tau^{2}}{\sigma}\right) \frac{1}{\tau}\left(\gamma-\tau+\frac{\tau^{2}}{\sigma}\right)$ & + & $-{ }^{3}$ & $-{ }^{4}$ & $+^{5}$ \\
$C S^{*}$ & $=\frac{1}{2 \tau}\left(\gamma-\tau+\frac{\tau^{2}}{\sigma}\right)^{2}$ & 0 & + & $+/-^{6}$ & - \\
$W^{*}$ & $=\left(\beta-\frac{1}{2}\left(\gamma-\tau+\frac{\tau^{2}}{\sigma}\right)\right) \frac{1}{\tau}\left(\gamma-\tau+\frac{\tau^{2}}{\sigma}\right)$ & + & $+^{7}$ & $-{ }^{8}$ & $-{ }^{9}$ \\
\hline
\end{tabular}

1. $\operatorname{sign}\left(\frac{\partial p^{*}}{\partial \tau}\right)=\operatorname{sign}\left(1-\frac{2 \tau}{\sigma}\right)=\operatorname{sign}(\sigma-2 \tau)$. We have found parameter values compatible with the conditions in Proposition 6 that support a positive sign as well as a negative one.

2. $\frac{\partial s^{*}}{\partial \tau}=-\frac{\gamma}{\tau^{2}}+\frac{1}{\sigma}<0$, since $\gamma \sigma>\tau^{2}$.

3. $\frac{\partial \pi^{*}}{\partial \gamma}=\frac{1}{\tau}\left(\beta-2\left(\gamma-\tau+\frac{\tau^{2}}{\sigma}\right)\right)<0$, since one of the conditions in Proposition 6 is $\beta \leq$ $2\left(\gamma-\tau+\frac{\tau^{2}}{\sigma}\right)$.

4. $\frac{\partial \pi^{*}}{\partial \tau}=\ldots=-\frac{1}{\tau^{2}}\left(\beta-\gamma+\frac{\tau^{2}}{\sigma}\right)\left(\gamma-\tau+\frac{\tau^{2}}{\sigma}\right)+\frac{1}{\tau^{2}}\left(\beta-\gamma+\tau-\frac{\tau^{2}}{\sigma}\right)\left(-\tau+2 \frac{\tau^{2}}{\sigma}\right)=\ldots=$ $-\left\{\frac{1}{\tau^{2}}(\beta-\gamma) \gamma+\frac{1}{\sigma}\left[2\left(\gamma-\tau+\frac{\tau^{2}}{\sigma}\right)-\beta\right]+\left(\frac{\tau}{\sigma}-1\right)^{2}\right\}<0$, since $\beta>\gamma$ and $\beta<2\left(\gamma-\tau+\frac{\tau^{2}}{\sigma}\right)$.

5. $\frac{\partial \pi^{*}}{\partial \sigma}=\frac{\tau}{\sigma^{2}}\left(2\left(\gamma-\tau+\frac{\tau^{2}}{\sigma}\right)-\beta\right)>0$, again, using the condition in Proposition 6 .

6. We have found parameter values compatible with the conditions in Proposition 6 that support a positive sign as well as a negative one. 
7. $\frac{\partial W^{*}}{\partial \gamma}=\frac{1}{\tau}\left(\beta-\gamma+\tau-\frac{\tau^{2}}{\sigma}\right)>0$.

8. As a result of an increase in $\tau$, the demand curve shifts inward. Moreover, the number of consumers decreases as a result of the increase in $\tau$. Both effects induce a decrease in welfare. In formulas, $W=\int_{x=0}^{\frac{1}{\tau}\left(\gamma-\tau+\frac{\tau^{2}}{\sigma}\right)}(\beta-\tau x) d x$. As $\tau$ increases, $\frac{1}{\tau}\left(\gamma-\tau+\frac{\tau^{2}}{\sigma}\right)$ decreases, and for each $x, \beta-\tau x$ decreases. Since for each $x$ in the domain of integration $\beta-\tau x$ is positive, it follows that $W$ decreases as $\tau$ increases.

9. $\frac{\partial W^{*}}{\partial \sigma}=-\frac{\tau}{\sigma^{2}}\left(\beta-\gamma+\tau-\frac{\tau^{2}}{\sigma}\right)<0$.

\section{C.5 Co-existence}

\begin{tabular}{llccccc}
\hline & & $\beta$ & $\gamma$ & $\tau$ & $\sigma$ \\
\hline$p^{*}$ & $=\frac{2}{9 \sigma}(2 \tau-\sqrt{\delta})(\tau+\sqrt{\delta})$ & $+^{1}$ & $-{ }^{2}$ & $+^{3}$ & $-{ }^{4}$ \\
$s^{*}$ & $=\frac{1}{3 \sigma}(2 \tau-\sqrt{\delta})$ & & + & - & $-{ }^{5}$ & $+^{6}$ \\
$n^{*}$ & $=1-\frac{1}{3 \sigma}(2 \tau-\sqrt{\delta})$ & - & + & + & - \\
$\pi^{*}$ & $=\frac{2}{27 \sigma^{2}}(2 \tau-\sqrt{\delta})^{2}(\tau+\sqrt{\delta})$ & $+^{7}$ & $-{ }^{8}$ & $+^{9}$ & $+^{10}$ \\
$C S^{*}$ & $=\gamma-\frac{1}{2} \tau-\frac{1}{9 \sigma^{2}}(2 \tau-\sqrt{\delta})^{2}(\sigma-\tau)$ & - & + & $-{ }^{11}$ & $-{ }^{12}$ \\
$W^{*}$ & $=\gamma-\frac{1}{2} \tau-\frac{1}{27 \sigma^{2}}(2 \tau-\sqrt{\delta})^{2}(3 \sigma-5 \tau-2 \sqrt{\delta})$ & & $+{ }^{13}$ & $+^{14}$ & $-{ }^{15}$ & $-{ }^{16}$ \\
\hline & where $\delta=4 \tau^{2}-3 \sigma(\beta-\gamma+\tau)$ & & & & \\
\hline
\end{tabular}

Notice that $C S^{*}=\left[\beta-\frac{1}{2} \tau s^{*}-p^{*}\right] s^{*}+\left[\gamma-\frac{1}{2} \tau n^{*}-\sigma\left(1-n^{*}\right)^{2}\right] n^{*}$.

Claim. The conditions in Proposition 7 imply $\sigma \in\left(\tau, \frac{4}{3} \tau\right)$ and $\sqrt{\delta} \in\left(\frac{1}{2} \tau, \tau\right)$.

Proof. The properties $\sigma \in\left(\tau, \frac{4}{3} \tau\right)$ and $\sqrt{\delta}<\tau$ follow directly from $\sigma, \beta>\gamma>\tau$ and $\delta \geq 0$. Left to be shown is $\sqrt{\delta}>\frac{1}{2} \tau$. First notice that

$$
\pi_{[S]}^{*}=\frac{1}{3 \sigma}(\tau+\sqrt{\delta})(\tau-\sqrt{\delta}) \quad \text { and } \quad \pi_{[S / \emptyset]}^{*}=\frac{1}{3 \sigma}(\tau+\sqrt{\delta})(\tau-\sqrt{\delta}) \frac{1}{\tau}\left(\gamma-\tau+\frac{\tau^{2}}{\sigma}\right) .
$$

Consider the case $\gamma \geq 2 \tau-\frac{\tau^{2}}{\sigma}$. Since $\pi^{*}>\pi_{[S]}^{*}$ we have

$$
2 \delta+(9 \sigma-8 \tau) \sqrt{\delta}-(9 \sigma-8 \tau) \tau>0 .
$$

Since this inequality is quadratic in $\sqrt{\delta}$ it is easily shown that it is satisfied if and only if

$$
\sqrt{\delta}>-\frac{1}{4}(9 \sigma-8 \tau)+\frac{1}{4} \sqrt{9 \sigma(9 \sigma-8 \tau)} .
$$

Since

$$
-\frac{1}{4}(9 \sigma-8 \tau)+\frac{1}{4} \sqrt{9 \sigma(9 \sigma-8 \tau)} \geq \frac{1}{2} \tau \quad \Longleftrightarrow \quad \sqrt{\sigma(9 \sigma-8 \tau)} \geq 3 \sigma-2 \tau \quad \Longleftrightarrow \quad \sigma \geq \tau
$$

we have shown that $\sqrt{\delta}>\frac{1}{2} \tau$. 
Next, consider the case $\gamma<2 \tau-\frac{\tau^{2}}{\sigma}$. Since $\pi^{*}>\pi_{[S / \emptyset]}^{*}$ we have

$$
2 \delta+\frac{1}{\tau}\left(9 \sigma \gamma-9 \sigma \tau+\tau^{2}\right) \sqrt{\delta}-\left(9 \sigma \gamma-9 \sigma \tau+\tau^{2}\right)>0
$$

This inequality is satisfied if and only if

$$
\sqrt{\delta}>-\frac{1}{4 \tau}\left(9 \sigma \gamma-9 \sigma \tau+\tau^{2}\right)+\frac{1}{4 \tau} \sqrt{9\left(\sigma \gamma-\sigma \tau+\tau^{2}\right)\left(9 \sigma \gamma-9 \sigma \tau+\tau^{2}\right)} .
$$

Since

$$
-\frac{1}{4 \tau}\left(9 \sigma \gamma-9 \sigma \tau+\tau^{2}\right)+\frac{1}{4 \tau} \sqrt{9\left(\sigma \gamma-\sigma \tau+\tau^{2}\right)\left(9 \sigma \gamma-9 \sigma \tau+\tau^{2}\right)} \geq \frac{1}{2} \tau
$$

if and only if

$$
\sqrt{\left(\sigma \gamma-\sigma \tau+\tau^{2}\right)\left(9 \sigma \gamma-9 \sigma \tau+\tau^{2}\right)} \geq 3 \sigma \gamma-3 \sigma \tau+\tau^{2}
$$

which is the case if and only if $\gamma \geq \tau$, we have shown that $\sqrt{\delta}>\frac{1}{2} \tau$.

1. $\frac{\partial p^{*}}{\partial \beta}=\frac{2}{9 \sigma}(2 \tau-\sqrt{\delta}) \frac{d \sqrt{\delta}}{d \beta}-\frac{2}{9 \sigma}(\tau+\sqrt{\delta}) \frac{d \sqrt{\delta}}{d \beta}=\frac{2}{9 \sigma}(\tau-2 \sqrt{\delta}) \frac{1}{2} \frac{1}{\sqrt{\delta}}(-3 \sigma)=\frac{1}{3 \sqrt{\delta}}(2 \sqrt{\delta}-\tau)>0$.

2. $\frac{\partial p^{*}}{\partial \gamma}=-\frac{\partial p^{*}}{\partial \beta}<0$.

3. $\frac{\partial p^{*}}{\partial \tau}=\frac{2}{9 \sigma}(2 \tau-\sqrt{\delta})\left(1+\frac{d \sqrt{\delta}}{d \tau}\right)+\frac{2}{9 \sigma}\left(2-\frac{d \sqrt{\delta}}{d \tau}\right)(\tau+\sqrt{\delta})=\frac{2}{9 \sigma}\left\{4 \tau+\sqrt{\delta}+\frac{d \sqrt{\delta}}{d \tau}(\tau-2 \sqrt{\delta})\right\}$ $=\frac{2}{9 \sigma} \frac{1}{\sqrt{\delta}}\left\{4 \tau \sqrt{\delta}+\delta+\frac{1}{2}(8 \tau-3 \sigma)(\tau-2 \sqrt{\delta})\right\}=\frac{2}{9 \sigma} \frac{1}{\sqrt{\delta}}\left\{\delta+\sqrt{\delta}(3 \sigma-4 \tau)+\left(4 \tau^{2}-\frac{3}{2} \sigma \tau\right)\right\}>$ $\frac{2}{9 \sigma} \frac{1}{\sqrt{\delta}}\left\{\delta-\tau^{2}+2 \tau^{2}\right\}>0$.

4. Notice that $\frac{d \sqrt{\delta}}{d \sigma}=\frac{1}{2} \frac{1}{\sqrt{\delta}}[-3(\beta-\gamma+\tau)]=-\frac{1}{2} \frac{1}{\sigma} \frac{1}{\sqrt{\delta}}\left[4 \tau^{2}-\delta\right]=-\frac{1}{2} \frac{1}{\sigma} \frac{1}{\sqrt{\delta}}(2 \tau-\sqrt{\delta})(2 \tau+\sqrt{\delta})$. Then $\frac{\partial p^{*}}{\partial \sigma}=\ldots=-\frac{2}{9 \sigma^{2}}(2 \tau-\sqrt{\delta})(\tau+\sqrt{\delta})-\frac{2}{9 \sigma^{2}}(\tau-2 \sqrt{\delta}) \frac{1}{2} \frac{1}{\sqrt{\delta}}(2 \tau-\sqrt{\delta})(2 \tau+\sqrt{\delta})=$ $\ldots=-\frac{1}{9 \sigma^{2}}(2 \tau-\sqrt{\delta})^{2} \frac{1}{\sqrt{\delta}} \tau<0$.

5. $\frac{\partial s^{*}}{\partial \tau}=\frac{1}{3 \sigma} \frac{1}{\sqrt{\delta}}\left(2 \sqrt{\delta}-4 \tau+\frac{3}{2} \sigma\right)<\frac{1}{3 \sigma} \frac{1}{\sqrt{\delta}}\left(2 \sqrt{\delta}-4 \tau+\frac{3}{2} \frac{4}{3} \tau\right)=\frac{1}{3 \sigma} \frac{1}{\sqrt{\delta}} 2(\sqrt{\delta}-\tau)<0$.

6. $\frac{\partial s^{*}}{\partial \sigma}=\ldots=-\frac{1}{3 \sigma^{2}}(2 \tau-\sqrt{\delta})+\frac{1}{6 \sigma^{2}} \frac{1}{\sqrt{\delta}}(2 \tau-\sqrt{\delta})(2 \tau+\sqrt{\delta})=\frac{1}{6 \sigma^{2}} \frac{1}{\sqrt{\delta}}(2 \tau-\sqrt{\delta})^{2}>0$.

7. $\frac{\partial \pi^{*}}{\partial \beta}=\frac{2}{27 \sigma^{2}}\left\{2(2 \tau-\sqrt{\delta})\left(-\frac{d \sqrt{\delta}}{d \beta}\right)(\tau+\sqrt{\delta})+(2 \tau-\sqrt{\delta})^{2} \frac{d \sqrt{\delta}}{d \beta}\right\}=-\frac{2}{9 \sigma^{2}}(2 \tau-\sqrt{\delta}) \sqrt{\delta} \frac{d \sqrt{\delta}}{d \beta}=$ $\frac{1}{3 \sigma}(2 \tau-\sqrt{\delta})>0$.

8. $\frac{\partial \pi^{*}}{\partial \gamma}=-\frac{\partial \pi^{*}}{\partial \beta}<0$.

9. $\frac{\partial \pi^{*}}{\partial \tau}=\frac{2}{27 \sigma^{2}}(2 \tau-\sqrt{\delta})\left\{2(\tau+\sqrt{\delta})\left[2-\frac{d \sqrt{\delta}}{d \tau}\right]+(2 \tau-\sqrt{\delta})\left[1+\frac{d \sqrt{\delta}}{d \tau}\right]\right\}=\ldots=\frac{2}{9 \sigma^{2}}(2 \tau-$ $\sqrt{\delta})\left\{\frac{3}{2}(\sigma-\tau)+\frac{1}{2}(2 \sqrt{\delta}-\tau)\right\}>0$. 
10. $\frac{\partial \pi^{*}}{\partial \sigma}=\frac{2}{27 \sigma^{2}}(2 \tau-\sqrt{\delta})\left\{-\frac{2}{\sigma}(2 \tau-\sqrt{\delta})(\tau+\sqrt{\delta})-2(\tau+\sqrt{\delta}) \frac{d \sqrt{\delta}}{d \sigma}+(2 \tau-\sqrt{\delta}) \frac{d \sqrt{\delta}}{d \sigma}\right\}=$ $\ldots($ see 4 . $) \ldots=\frac{2}{27 \sigma^{2}}(2 \tau-\sqrt{\delta})\left\{-\frac{2}{\sigma}(2 \tau-\sqrt{\delta})(\tau+\sqrt{\delta})+\frac{3}{2 \sigma}(2 \tau-\sqrt{\delta})(2 \tau+\sqrt{\delta})\right\}=$ $\ldots=\frac{1}{27 \sigma^{3}}(2 \tau-\sqrt{\delta})^{3}>0$.

11. $\frac{\partial C S^{*}}{\partial \tau}=\frac{\partial W^{*}}{\partial \tau}-\frac{\partial \pi^{*}}{\partial \tau}<0$ (using 9. and 15.).

12. $\frac{\partial C S^{*}}{\partial \sigma}=\frac{\partial W^{*}}{\partial \sigma}-\frac{\partial \pi^{*}}{\partial \sigma}<0$ (using 10. and 16.).

13. $\frac{\partial W^{*}}{\partial \beta}=-\frac{1}{27 \sigma^{2}}(2 \tau-\sqrt{\delta})\left\{2\left(-\frac{d \sqrt{\delta}}{d \beta}\right)(3 \sigma-5 \tau-2 \sqrt{\delta})+(2 \tau-\sqrt{\delta})\left(-2 \frac{d \sqrt{\delta}}{d \beta}\right)\right\}=\ldots=\frac{1}{3 \sigma}(2 \tau-$ $\sqrt{\delta}) \frac{1}{\sqrt{\delta}}(\tau+\sqrt{\delta}-\sigma)>\frac{1}{3 \sigma}(2 \tau-\sqrt{\delta}) \frac{1}{\sqrt{\delta}} \frac{1}{6} \tau>0$.

14. $\frac{\partial W^{*}}{\partial \gamma}=1-\frac{1}{27 \sigma^{2}}(2 \tau-\sqrt{\delta})\left\{2\left(-\frac{d \sqrt{\delta}}{d \gamma}\right)(3 \sigma-5 \tau-2 \sqrt{\delta})+(2 \tau-\sqrt{\delta})\left(-2 \frac{d \sqrt{\delta}}{d \gamma}\right)\right\}=\ldots=$ $1-\frac{1}{3 \sigma}(2 \tau-\sqrt{\delta}) \frac{1}{\sqrt{\delta}}(\tau+\sqrt{\delta}-\sigma)=\frac{1}{3 \sigma} \frac{1}{\sqrt{\delta}}(\tau+\sqrt{\delta})[2(\sigma-\tau)+\sqrt{\delta}]>0$.

15. $\frac{\partial W^{*}}{\partial \tau}=\ldots=-\frac{1}{2}-\frac{1}{9}(2 \tau-\sqrt{\delta})\left\{\frac{1}{\sigma}-\frac{2 \tau}{\sigma^{2}}-\frac{\sqrt{\delta}}{\sigma^{2}}+\frac{8 \tau^{2}}{\sigma^{2} \sqrt{\delta}}-\frac{11 \tau}{\sigma \sqrt{\delta}}+\frac{3}{\sqrt{\delta}}\right\}$. First, let $\sigma=a \tau$ for $a \in\left(1, \frac{4}{3}\right)$. Then $\frac{\partial W^{*}}{\partial \tau}=-\frac{1}{2}-\frac{1}{9}(2 \tau-\sqrt{\delta})\left\{\frac{1}{a \tau}-\frac{2}{a^{2} \tau}-\frac{\sqrt{\delta}}{a^{2} \tau^{2}}+\frac{1}{\sqrt{\delta}}\left(\frac{8}{a^{2}}-\frac{11}{a}+3\right)\right\}$. Next, define $\alpha \equiv \frac{1}{a \tau}-\frac{2}{a^{2} \tau}-\frac{\sqrt{\delta}}{a^{2} \tau^{2}}+\frac{1}{\sqrt{\delta}}\left(\frac{8}{a^{2}}-\frac{11}{a}+3\right)$. If $\alpha \geq 0$ then clearly $\frac{\partial W^{*}}{\partial \tau}<0$. Suppose $\alpha<0$. Then $\alpha>\frac{1}{a \tau}-\frac{2}{a^{2} \tau}-\frac{\tau}{a^{2} \tau^{2}}+\frac{1}{\frac{1}{2} \tau}\left(\frac{8}{a^{2}}-\frac{11}{a}+3\right)=\frac{1}{\tau}\left\{\frac{13}{a^{2}}-\frac{21}{a}+6\right\}>\frac{1}{\tau} \min _{a}\left\{\frac{13}{a^{2}}-\frac{21}{a}+6\right\}=-\frac{129}{52 \tau}$, where for the first inequality it has to be noted that $\frac{8}{a^{2}}-\frac{11}{a}+3<0$ for $a \in\left(1, \frac{4}{3}\right)$. Hence $\frac{\partial W^{*}}{\partial \tau}=-\frac{1}{2}-\frac{1}{9}(2 \tau-\sqrt{\delta}) \cdot \alpha<-\frac{1}{2}-\frac{1}{9} \frac{3}{2} \tau \cdot\left(-\frac{129}{52 \tau}\right)=-\frac{9}{104}<0$.

16. $\frac{\partial W^{*}}{\partial \sigma}=\frac{2}{27 \sigma^{3}}(2 \tau-\sqrt{\delta})^{2}(3 \sigma-5 \tau-2 \sqrt{\delta})-\frac{2}{27 \sigma^{2}}(2 \tau-\sqrt{\delta})\left(-\frac{d \sqrt{\delta}}{d \sigma}\right)(3 \sigma-5 \tau-2 \sqrt{\delta})-\frac{1}{27 \sigma^{2}}(2 \tau-$ $\sqrt{\delta})^{2}\left[3-2 \frac{d \sqrt{\delta}}{d \sigma}\right]=\ldots=\frac{1}{27 \sigma^{3}}(2 \tau-\sqrt{\delta})^{2} \frac{1}{\sqrt{\delta}}\{2 \sqrt{\delta}(3 \sigma-5 \tau-2 \sqrt{\delta})-(2 \tau+\sqrt{\delta})(3 \sigma-5 \tau-2 \sqrt{\delta})-$ $3 \sigma \sqrt{\delta}-(2 \tau-\sqrt{\delta})(2 \tau+\sqrt{\delta})\}=\ldots=-\frac{1}{27 \sigma^{3}}(2 \tau-\sqrt{\delta})^{2} \frac{1}{\sqrt{\delta}}\{6 \tau(\sigma-\tau)+\sqrt{\delta}(\tau+\sqrt{\delta})\}<0$. 